Article

\title{
Intrusion of Saline Water into a Coastal Aquifer Containing Palaeogroundwater in the Viimsi Peninsula in Estonia
}

\author{
Valle Raidla 1,*(D), Joonas Pärn 1,2, Werner Aeschbach ${ }^{3} \mathbb{D}$, György Czuppon 4,5 , Jüri Ivask ${ }^{2}$, \\ Madis Kiisk ${ }^{6}$, Robert Mokrik ${ }^{7}$, Vytautas Samalavičius ${ }^{7}$, Siiri Suursoo ${ }^{6}$, Siim Tarros ${ }^{1}$ and \\ Therese Weissbach ${ }^{3}$ \\ 1 Department of Hydrogeology and Environmental Geology, Geological Survey of Estonia, Kreutzwaldi 5, \\ 44314 Rakvere, Estonia; joonas.parn@egt.ee (J.P.); siim.tarros@egt.ee (S.T.) \\ 2 Institute of Geology, Tallinn University of Technology, Ehitajate tee 5, 12616 Tallinn, Estonia; \\ Juri.Ivask@ttu.ee \\ 3 Institute of Environmental Physics, University of Heidelberg, Im Neuenheimer Feld 229, \\ D-69120 Heidelberg, Germany; aeschbach@iup.uni-heidelberg.de (W.A.); \\ Therese.Weissbach@iup.uni-heidelberg.de (T.W.) \\ 4 Institute for Geological and Geochemical Research, Research Centre for Astronomy and Earth Sciences, \\ Hungarian Academy of Sciences, Budaörsi út 45., H-1112 Budapest, Hungary; czuppon@geochem.hu \\ 5 Department of Hydrogeology and Engineering Geology, University of Miskolc, \\ 3515 Miskolc-Egyetemváros, Hungary \\ 6 Institute of Physics, University of Tartu, W. Ostwaldi Str. 1, 50411 Tartu, Estonia; madis.kiisk@ut.ee (M.K.); \\ siiri.suursoo@ut.ee (S.S.) \\ 7 Department of Hydrogeology and Engineering Geology, Vilnius University, M.K. Čiurlionio str. 21/27, \\ LT-03101 Vilnius, Lithuania; robert.mokrik@gf.vu.lt (R.M.); vytautas.samalavicius@chgf.vu.lt (V.S.) \\ * Correspondence: valle.raidla@egt.ee
}

Received: 14 November 2018; Accepted: 14 January 2019; Published: 17 January 2019

\begin{abstract}
The Viimsi peninsula is located north-east of Tallinn, capital of Estonia. The Cambrian-Vendian $(\mathrm{Cm}-\mathrm{V})$ aquifer system is a sole source of drinking water in the area. Historically, the groundwater exploitation has led to freshening of groundwater in the peninsula, but in recent years an increase in chloride concentrations and enrichment in $\delta^{18} \mathrm{O}$ values has been detected, but in recent years hydrochemical parameters indicate an increasing influence of a saline water source. The exact origin of this saline water has remained unclear. The aim of the current study is to elucidate whether the increase in $\mathrm{Cl}^{-}$concentrations is related to seawater intrusion or to the infiltration of saline water from the underlying crystalline basement. To identify the source of salinity, chemical composition of the groundwater and the isotope tracers (e.g., $\delta^{18} \mathrm{O}$ and radium isotopes) were studied in the Viimsi peninsula in the period from 1987 to 2018. Our results show that chemical composition of $\mathrm{Cm}-\mathrm{V}$ groundwater in the peninsula is clearly controlled by three-component mixing between glacial palaeogroundwater, saline water from the underling crystalline basement and modern meteoric water. The concentrations of Ra are also significantly affected by the mixing, but the spatial variation of radium isotopes $\left({ }^{226} \mathrm{Ra}\right.$ and $\left.{ }^{228} \mathrm{Ra}\right)$ suggests the widespread occurrence of the $U$ in the surrounding sedimentary sequence. Our hypothesis is that, in addition to $U$ originating from the crystalline basement, some $U$ could be associated with secondary $U$ deposits in sedimentary rocks. The formation of these secondary $U$ deposits could be related to glacial meltwater intrusion in the Pleistocene. Although the results suggest that the infiltration of saline groundwater from the underlying crystalline basement as the main source of salinity in the study area, the risk of seawater intrusion in the future cannot be ruled out. It needs to be highlighted that the present groundwater monitoring networks may not be precise enough to detect the potential seawater intrusion and subsequent changes in water quality of the $\mathrm{Cm}-\mathrm{V}$ aquifer system in the Viimsi peninsula.
\end{abstract}


Keywords: mixing; salinisation; oxygen isotopes; radium isotopes

\section{Introduction}

The growing urbanisation in coastal areas rises the demand for water; an increase in groundwater abstraction can lead to a subsequent lowering of groundwater levels and deterioration in groundwater quality. One of the reasons for decline in water quality is groundwater salinisation through intrusion of seawater or relict saline groundwater from adjacent aquifers [1-6]. These processes could initiate chemical reactions between the aquifer-forming rocks and groundwater, when the composition of groundwater is changed by mixing between the intruding saltwater and fresh groundwater originally occupying a coastal aquifer [6-8]. The geochemical properties of rocks could determine the dominant chemical processes during mixing [6,9-11]. Clay minerals and organic matter can adsorb/desorb cations while dissolution/precipitation processes can occur in case carbonate minerals are present in the aquifer matrix [2,12-17].

Although Viimsi peninsula is next to an urbanised area, local infrastructure has stayed relatively isolated and the community uses groundwater for water supply. During the last decade, that population has tripled, due to active real estate development and is now 19,000 with a prospect of soon reaching 30,000 inhabitants. For its drinking water, the region relies on just one aquifer system, the Cambrian-Vendian $(\mathrm{Cm}-\mathrm{V})$. The groundwater from the $\mathrm{Cm}-\mathrm{V}$ aquifer system in North Estonia is typically strongly depleted in ${ }^{18} \mathrm{O}\left(\delta^{18} \mathrm{O}=-18\right.$ to $-23 \%$ ) [18] with respect to local shallow groundwater $(-11$ to $-12.5 \%$ ) [19]. The $\mathrm{Cm}-\mathrm{V}$ groundwater originates from glacial meltwater of the Scandinavian Ice Sheet that covered the territory of Estonia during the Pleistocene $[20,21]$. Thus, the aquifer system contains groundwater that is essentially non-renewable on a human timescale and the quality of which can be strongly altered during groundwater abstraction.

In the past 60 years, the $\mathrm{Cm}-\mathrm{V}$ aquifer system has extensively been used for public water supply on the Viimsi peninsula. Such groundwater exploitation led to the drawdown of hydraulic heads about 15 metres below the pre-development levels [22,23] and caused freshening of groundwater in the south-western part of the peninsula, where an ancient buried valley cuts through the aquitard confining the aquifer system. Modern meteoric waters can enter the $\mathrm{Cm}-\mathrm{V}$ aquifer system through this pathway once groundwater abstraction reduces the piezometric levels in the aquifer system. In the past 25 years, the groundwater consumption has decreased and piezometric levels have slowly recovered, but the recent rapid increase in population on the peninsula has once again raised groundwater consumption. The new decrease in the piezometric levels has led to an increase in salinity in the deeper part of the aquifer system. This is exemplified by the rise in chloride concentrations and enrichment in stable oxygen isotope composition [24]. The exact origin of the saline water entering the aquifer system has remained unclear. Suursoo et al. [24] suggested three possible origins for the increased salinity in the aquifer system: (1) Seawater intrusion through the aquifer's discharge area in the Gulf of Finland; (2) intrusion of relict saline water from the underlying crystalline aquifer and (3) combination of the former two.

Suursoo et al. [24] also observed that changes in salinity were related to changes in the activity of Ra isotopes in groundwater. Radium activity in the $\mathrm{Cm}-\mathrm{V}$ aquifer system has been attributed to the prolonged contact with radioactive rocks of the crystalline basement [25-27], where higher concentrations of Ra precursors, $U$ and Th have been observed. However, several studies [28-30] have indicated that the behaviour of $R a$ is complicated and affected not only by the content of $U$ and $T h$ in the aquifer-forming rocks, but also by geochemical processes in the vicinity of Ra sources. Radium forms bivalent cations $\left(\mathrm{Ra}^{2+}\right)$ in natural environments under low salinity conditions that behave similarly to other divalent alkaline-earth cations, such as $\mathrm{Ca}^{2+}$ and $\mathrm{Mg}^{2+}$ [31]. Thus, adsorption/desorption processes are significant mobility controllers of Ra isotopes in groundwater, because Ra has to compete for adsorption sites with other divalent cations, known as the "competing ion effect" [30,32-35]. 
Furthermore, Ra may form low-solubility complexes with sulphates and carbonates, and thus be precipitate from the solution $[28,36-38]$.

The previous study on the same topic by Suursoo et al. [24] was based on a limited number of samples from the central part of the Viimsi peninsula. This contribution relies on a broader dataset have been collected from a much wider area of the peninsula. In our analysis, both previously published and unpublished data collected in the period from 1987 to 2018 have been used. This enabled us to study the effects that previous increases in groundwater abstraction have had on the chemical and isotopic composition of groundwater in the study area. Our main aim was to elucidate whether the current increase in $\mathrm{Cl}^{-}$concentration observed in the peninsula at present was related to seawater intrusion or rather to the intrusion of saline water from the underlying crystalline basement. The study also looked more specifically at the mechanisms of Ra mobilisation in the aquifer system. The aim was to clarify whether the Ra isotope activities could offer further insights into processes that have influenced the changes in groundwater quality in the Viimsi peninsula.

\section{Geology and Hydrogeological Setting}

The Viimsi peninsula is in northern Estonia, next to the republic's capital, Tallinn (Figure 1a). The surface area of the peninsula is about $50 \mathrm{~km}^{2}$. The landscape is flat, covered with glacial and marine deposits from the Quaternary Period. Except for the southern part of the peninsula that is underlain by Ordovician carbonate rocks, Quaternary deposits lie directly on Lower Cambrian clays and clay stones (the Lükati-Lontova regional aquitard) with a thickness of $\sim 40 \mathrm{~m}$ (Figure 1b). This formation confines the underlying $\mathrm{Cm}-\mathrm{V}$ aquifer system, which is the only major aquifer system in the study area. The $\mathrm{Cm}-\mathrm{V}$ aquifer system contains groundwater with low TDS that has the most depleted isotopic composition recorded in Europe $\left(\delta^{18} \mathrm{O}\right.$ values from $-18.5 \%$ to $\left.-23 \%\right)$, whereas the $\delta^{18} \mathrm{O}$ values of the modern groundwater range from -11 to $-12.5 \%$ [18-20].

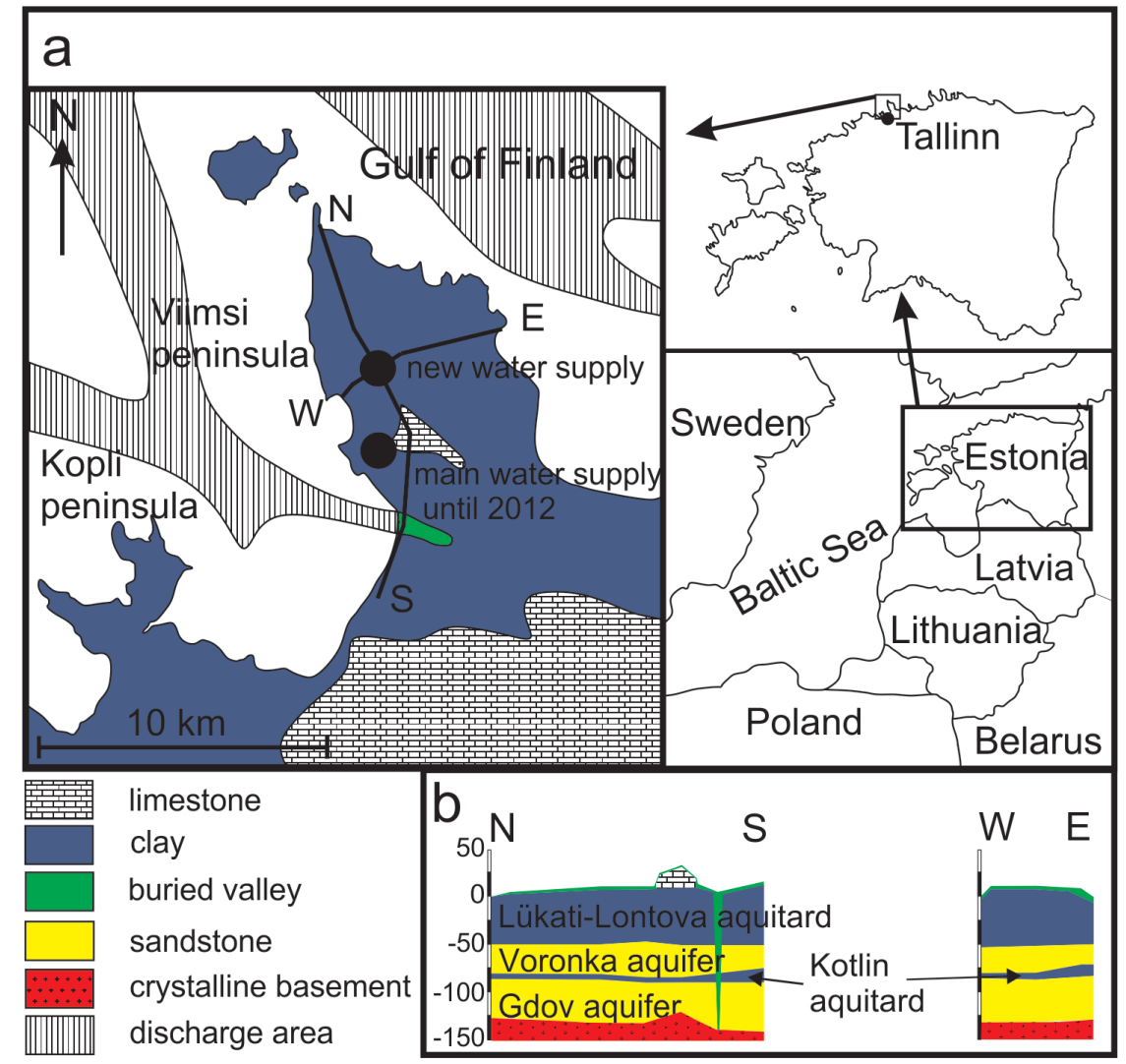

Figure 1. Geological scheme and location of the Viimsi peninsula in Estonia (a), the west-east and the north-south cross-sections of the peninsula (b) (modified after Suursoo et al. [24]). 
The water-bearing rocks of the $\mathrm{Cm}-\mathrm{V}$ aquifer consist of $\sim 70 \mathrm{~m}$ thick formation of silt- and sandstones of the Ediacaran age. These formations outcrop 1 to $3 \mathrm{~km}$ north of the peninsula coastline, at the bottom of the Gulf of Finland (Figure 1). Although the aquifer system is well confined from the intrusion of modern meteoric water and surface pollution by the $40 \mathrm{~m}$ thick Lontova-Lükati aquitard, the latter is cut through by deep pre-Quaternary buried valley filled with Quaternary sediments in the south-western margin of the peninsula. Here, the aquifer system is exposed to groundwater recharge from the modern meteoric water (Figure 1).

Although a clear aquitard layer is lacking inside the $\mathrm{Cm}-\mathrm{V}$ aquifer system in the peninsula, it is possible to distinguish between two stipulated aquifers-the upper Voronka $\left(\mathrm{V}_{2 \mathrm{vr}}\right)$ and the lower $G$ dov aquifer $\left(\mathrm{V}_{2 g d}\right)$ (Figure $\left.1 \mathrm{~b}\right)$. Lenses of a metre or two thick siltstones of low permeability inside the aquifer-forming rocks could be considered a conditional aquitard (the Kotlin aquitard) between the two aquifers. The Voronka aquifer is in the sandstones of the Lower-Cambrian (Lontova Formation) and Ediacaran ages. The Gdov aquifer is hosted by underlying sand- and siltstones of the Ediacaran age. Transmissivity between the aquifers varies, being from 40 to $1,950 \mathrm{~m}^{2} \cdot \mathrm{d}^{-1}$ in the Gdov and only from 26 to $330 \mathrm{~m}^{2} \cdot \mathrm{d}^{-1}$ in the Voronka aquifer [39].

The aquifers have a similar hydraulic head, but the chemical composition of the deeper and shallower parts of the aquifer system are fundamentally different. Groundwater in the Gdov aquifer is typically more saline than groundwater in the upper Voronka aquifer (TDS concentrations of $700 \mathrm{mg} \cdot \mathrm{L}^{-1}$ and $200 \mathrm{mg} \cdot \mathrm{L}^{-1}$, respectively) [24]. Variability in $\delta^{18} \mathrm{O}$ values is from -12 to $-22.5 \%$ in the Gdov and $-20 \pm 0.5 \%$ in the Voronka aquifer. Additionally, elevated radionuclide contents (mainly ${ }^{226} \mathrm{Ra}$ activity) have been detected in the Gdov aquifer [24].

The $\mathrm{Cm}-\mathrm{V}$ aquifer system lies on the weathered and fractured crystalline basement, which is composed of metamorphosed mafic rocks and alumino-gneisses [40,41], and forms the so-called crystalline basement aquifer. The information about the water in this aquifer is scarce, but available data show that it contains $\mathrm{Na}-\mathrm{Cl}$ or $\mathrm{Ca}-\mathrm{Cl}$ type brackish and saline groundwater with electrical conductivity from 2 to $20 \mathrm{mS} \cdot \mathrm{cm}^{-1}[25,42]$. Several studies [21,24,42] have shown that the $\mathrm{Cm}-\mathrm{V}$ aquifer system and the underlying crystalline basement aquifer are hydraulically connected and the hydrochemistry in the deeper Gdov aquifer system is influenced by saline water in the crystalline basement aquifer.

Groundwater abstraction on the Viimsi peninsula started in the early 1960s and groundwater consumption increased constantly until the beginning of 1990s. By 1991, the piezometric heads in the $\mathrm{Cm}-\mathrm{V}$ aquifer system had decreased to -7 to $-8 \mathrm{~m}$ b.s.l. and in the southern part of the peninsula occasionally even to $-16 \mathrm{~m}$ b.s.l. Since 1994 , when groundwater consumption started to decrease, and the hydraulic heads slowly recovered. This process proceeded more swiftly in the deeper-lying Gdov aquifer [43]. During the period after 1994, the groundwater was pumped from the wells which were scattered mainly over the south-western part of the peninsula. In 2012, a new water-supply station was opened, and the groundwater consumption was concentrated to 11 wells in the central part of the peninsula (Figure 1).

\section{Material and Methods}

In total, 91 groundwater samples were collected from 53 private and municipal water supply wells in 2011 and 2016 (Table 1) to be analysed for hydrochemical composition and for stable isotopes of water and radium. The wells in the main groundwater intake were sampled only in their active production stage to ensure that the wells were pumped at least 2 to 3 volumes before sampling.

Samples for hydrochemical analysis were filtered through a $0.45-\mu \mathrm{m}$ filter and cation samples were acidified to $\mathrm{pH} \sim 2$ to 3 using nitric acid. Major and minor ion concentrations were measured using DIONEX ICS-1100 ion chromatograph (manufacturer Thermo Fisher Scientific, USA) in Tallinn University of Technology (TalTech). The analytical precision for major and minor ionic components was \pm 0.5 to $2.6 \%$ and \pm 1.3 to $4.3 \%$, for cations and anions, respectively. 
For Ra measurements, groundwater from a tap was taken directly into a $5 \mathrm{~L}$ plastic canister. Immediately after sampling, water was acidified with concentrated $\mathrm{HNO}_{3}{ }^{-}$to $\mathrm{pH} 1$ to 2 . Measurements were performed at the University of Tartu. Radium was co-precipitated with $\mathrm{Ba}\left(\mathrm{SO}_{4}\right)_{2}$ and then the precipitate was put into an aluminium vessel and homogenised with $5 \mathrm{~g}$ of epoxy resin. Epoxy was added to minimise radon $\left({ }^{222} \mathrm{Rn}\right.$ and $\left.{ }^{220} \mathrm{Rn}\right)$ leakage from the sample [44]. The samples were measured after at least 3 weeks from sample collection on a high purity germanium gamma spectrometer (coaxial type detector GEM 35200 (manufacturer E\&G Ortec, USA), coaxial GCD 50200 or planar GPD 50400 (manufacturer BSI- Baltic Scientific Instruments, Latvia). The typical measurement time was about one day. ${ }^{226} \mathrm{Ra}$ was determined according to its daughter nuclide ${ }^{214} \mathrm{~Pb}$ (gamma peaks 242.00 , $295.22,351.93 \mathrm{keV}) .{ }^{228} \mathrm{Ra}$ was analysed via the gamma peaks of ${ }^{228} \mathrm{Ac}(338.32,911.20,968.96 \mathrm{keV})$. A calibration was performed with certified reference materials RGU-1 and RGTh-1 (IAEA-International Atomic Energy Agency). For that, calibration sources with similar geometry (a few grams of the reference material mixed with $5 \mathrm{~g}$ of epoxy resin in an aluminium vessel) were prepared and measured. Minimum detectable activity concentrations were $15 \mathrm{mBq} \cdot \mathrm{L}^{-1}$ and $20 \mathrm{mBq} \cdot \mathrm{L}^{-1}$ for ${ }^{226} \mathrm{Ra}$ and ${ }^{228} \mathrm{Ra}$, respectively.

The unfiltered samples for stable isotope analyses were poured into $15 \mathrm{~mL}$ HDPE bottles and stored in a cold, dark environment. Stable hydrogen and oxygen isotope measurements were performed at the Institute for Geological and Geochemical Research in Budapest (Hungary) using the liquid water isotope analyser (LWIA), manufactured by Los Gatos Research Ltd. (LWIA-24d) and in the laboratory of mass-spectrometry in the TalTech Department of Geology using a Picarro L2120-i Isotopic Water Analyser. The $\delta^{2} \mathrm{H}$ and $\delta^{18} \mathrm{O}$ values were determined simultaneously and expressed relative to VSMOW standard in \%o. Standardisation was conducted using international and laboratory standard water samples. The precisions were better than $\pm 1.0 \%$ and $\pm 0.15 \%$ o for hydrogen and oxygen, respectively, for samples measured in Budapest and better than $\pm 1.0 \%$ and $\pm 0.1 \%$ for samples measured in Tallinn.

Noble gas samples were collected into copper tubes fixed on aluminium racks [45] during two fieldwork campaigns in 2013 and 2015. These copper tubes contained about $20 \mathrm{~g}$ of water and were closed vacuum tight by stainless steel clamps. Noble gas contents were determined at the Institute of Environmental Physics at Heidelberg University with a GV Instruments MM 5400 mass spectrometer.

\section{Results and Discussion}

\subsection{Mixing}

The data for the isotopic, major cation and anion compositions of the sampled groundwater in the $\mathrm{Cm}-\mathrm{V}$ aquifer system in the Viimsi peninsula are given in Table 1 . The $\delta^{18} \mathrm{O}$ and $\delta^{2} \mathrm{H}$ values of groundwater vary from -11.7 to $-22.8 \%$ and from -84 to $-170 \%$, respectively, and plot close to the Global Meteoric Water Line (GMWL) (Figure 2). Chemical and isotopic composition varies both laterally and with depth. The $\delta^{18} \mathrm{O}$ values and $\mathrm{Cl}^{-}$concentrations of the lower Gdov aquifer are from -12 to -22.5 and 10 to $730 \mathrm{mg} \cdot \mathrm{L}^{-1}$, respectively. The isotopic composition of groundwater is more uniform in the upper Voronka aquifer $\left(\delta^{18} \mathrm{O} \approx-20.5 \% 0\right)$ and $\mathrm{Cl}^{-}$content there is no higher than $80 \mathrm{mg} \cdot \mathrm{L}^{-1}$. 
Table 1. Chemical composition of the $\mathrm{Cm}-\mathrm{V}$ groundwaters in the Viimsi peninsula. Bdl-below detection limit. Previously published data is referred to as: 1-Yezhova et al. [46]; 2—Savitskaja et al. [47]; 3—Savitskaja and Viigand [25]; 4—Vaikmäe et al. [20]; 5—Raidla et al. [18]; 6—Raidla et al. [21]; 7—Raidla et al. [48]; 8 -Suursoo et al. [24].

\begin{tabular}{|c|c|c|c|c|c|c|c|c|c|c|c|c|c|c|c|c|c|c|c|c|c|c|c|c|c|}
\hline No & $\begin{array}{c}\text { Well } \\
\text { ID }\end{array}$ & Date & $\begin{array}{c}\text { Depth } \\
\text { m }\end{array}$ & $\mathrm{pH}$ & $\begin{array}{c}\text { EC } \\
\mu \mathrm{S} \cdot \mathrm{cm}^{-}\end{array}$ & $\begin{array}{r}\mathrm{T} \\
1{ }^{\circ} \mathrm{C} \\
\end{array}$ & $\begin{array}{l}\delta^{18} \mathrm{O} \\
\% \text { VSN } \\
\end{array}$ & $\begin{array}{r}\delta^{2} \mathrm{H} \\
\text { MOW }\end{array}$ & $\begin{array}{r}{ }^{226} \mathrm{Ra} \\
\mathrm{Bq}\end{array}$ & $\mathrm{L}^{-128} \mathrm{Ra}$ & $\mathrm{Ca}^{2+}$ & $\mathrm{Mg}^{2+}$ & $\mathrm{Na}^{+}$ & $\mathrm{K}^{+}$ & $\mathrm{HCO}_{3}{ }^{-}$ & $\mathrm{Cl}^{-}$ & $\mathrm{SO}_{4}{ }^{2-}$ & $\begin{array}{c}\mathrm{Br}^{-} \\
\mathrm{mg} \cdot \mathrm{L}^{-1}\end{array}$ & $\mathrm{Ba}^{2+}$ & $\mathrm{Mn}^{2+}$ & $\mathrm{NH}_{4}{ }^{+}$ & $\mathrm{NO}_{3}{ }^{-}$ & $\mathrm{Sr}^{2+}$ & $\mathrm{Fe}^{2+}$ & $\mathrm{F}^{-}$ \\
\hline \multicolumn{26}{|c|}{ Voronka aquifer $\left(\mathrm{V}_{2 \mathrm{vr}}\right)$} \\
\hline $1^{2}$ & 124 & 01.06.1988 & 101.5 & & & & -21.5 & & & & & & & & & & & & & & & & & & \\
\hline $2^{5}$ & 124 & 13.04 .2005 & 101.5 & 8.0 & 411 & 8.4 & -20.8 & & 0.16 & 0.10 & 34.5 & 10.1 & 34.7 & 7.3 & 146.4 & 56.7 & bdl & 0.2 & bdl & & & 0.07 & & 0.18 & \\
\hline 3 & 158 & 26.06 .1990 & 110 & 7.6 & & & & & & & 102.1 & 21.3 & 79.1 & 1.0 & 311.2 & 12.8 & 33.3 & & & 0.03 & bdl & 12.10 & & & \\
\hline 4 & 158 & 28.10 .2014 & 110 & & & & -14.9 & -107.9 & & & & & & & & & & & & & & & & & \\
\hline 5 & 171 & 08.10 .2014 & 95 & 8.2 & 461 & 8.4 & -20.7 & -157.2 & 0.11 & 0.30 & 44.7 & 13.5 & 29.6 & 8.8 & 170.8 & 53.3 & 0.7 & bdl & bdl & 0.41 & & & & & 0.47 \\
\hline $6^{5}$ & 179 & 12.04 .2005 & 90 & 7.8 & 364 & 8.2 & -20.3 & & 0.40 & 0.16 & 30.9 & 13.6 & 28.0 & 8.7 & 183.1 & 41.1 & bdl & 0.7 & bdl & & & 0.05 & & 0.13 & \\
\hline 7 & 179 & 09.06.2014 & 90 & 8.1 & 405 & 8.1 & -20.5 & -154.6 & 0.07 & 0.20 & 35.9 & 13.2 & 28.1 & 8.5 & & 36.5 & 0.1 & bdl & 0.37 & 0.27 & 0.17 & & 0.63 & & 0.49 \\
\hline $8^{2}$ & 185 & 11.08.1991 & 95 & & & & -20.4 & & & & & & & & & & & & & & & & & & \\
\hline $9^{5}$ & 187 & 13.04 .2005 & 70 & 7.8 & 254 & 8.3 & -21.3 & & 0.13 & 0.10 & 22.6 & 8.4 & 15.0 & 6.0 & 152.5 & 11.0 & bdl & 0.6 & bdl & & & 0.05 & & 0.08 & \\
\hline 10 & 187 & 13.11 .2013 & 70 & 8.0 & 136 & 8.5 & -21.3 & -161.1 & 0.11 & 0.25 & 21.9 & 3.7 & 11.7 & 4.2 & 155.6 & 6.7 & 0.2 & & 1.2 & 0.08 & 0.10 & 0.01 & 0.23 & 0.17 & 0.29 \\
\hline $11^{1}$ & 509 & 05.12 .1989 & & & & & & & & & 44.1 & 21.9 & 42.6 & & 220.0 & 80.0 & 1.2 & & & & 0.40 & & & & \\
\hline $12^{2}$ & 1153 & 04.07.1991 & 77.5 & & & & -21.8 & & & & & & & & & & & & & & & & & & \\
\hline $13^{3}$ & 1153 & 05.07.1994 & 77.5 & 7.4 & & & -21.4 & & & & 23.2 & 10.0 & 18.2 & 7.0 & 158.7 & 20.6 & 5.9 & & & & & & & & \\
\hline $14^{4}$ & 1153 & 30.08 .1995 & 77.5 & & & & -21.5 & & & & 36.1 & 32.7 & & & 128.1 & 81.0 & 13.0 & & & & & & & & \\
\hline $15^{5}$ & 1153 & 13.04 .2005 & 77.5 & 7.8 & 253 & 8.5 & -21.5 & & bdl & 0.11 & 24.6 & 7.2 & 16.7 & 7.0 & 140.3 & 8.5 & bdl & 0.7 & bdl & & & 0.07 & & 0.14 & \\
\hline $16^{4}$ & 8914 & 30.08 .1995 & 75 & & & & -20.0 & & & & & & & & & & & & & & & & & & \\
\hline $17^{6}$ & 8914 & 12.08 .1996 & 75 & & & & -20.3 & & & & & & & & & & & & & & & & & & \\
\hline 18 & 8914 & 06.09 .2013 & 75 & 8.1 & 262 & 9.5 & & & 0.24 & 0.36 & 30.7 & 11.7 & 25.1 & 6.4 & 170.8 & 36.7 & bdl & & bdl & 0.39 & 0.17 & 0.04 & 0.40 & bdl & 0.31 \\
\hline 19 & 8914 & 02.09 .2016 & 75 & 8.0 & 386 & 9.1 & -20.6 & -153.7 & & & 33 & 12.0 & 250 & 8.2 & 163.5 & 38.0 & bll & bdl & b & bdl & 0.22 & & 0.49 & & 0.65 \\
\hline 20 & 11570 & 01.07 .2016 & 100 & 8. & 649 & 9.1 & -21.4 & -161.0 & & & 37.0 & 8.3 & 20.0 & 5.6 & 109.0 & 68.0 & 14.0 & 0.7 & bdl & 0.60 & 0.12 & & 0.14 & & 0.45 \\
\hline $21^{5}$ & 19570 & 13.04 .2005 & 109 & 8.0 & 700 & 8.4 & -17.7 & & 0.39 & 0.31 & 59.9 & 13.7 & 64.0 & 8.8 & 195.2 & 134.0 & bdl & 0.4 & bdl & & & 0.07 & & 0.07 & \\
\hline $22^{8}$ & 23886 & 09.09 .2016 & 124.5 & 8.2 & 1366 & 8.8 & -20.6 & -152.1 & & & 28.0 & 9.9 & 18.0 & 7.6 & 141.7 & 14.0 & bdl & bdl & bdl & bdl & 0.20 & & 0.45 & & 0.51 \\
\hline $25^{8}$ & 25687 & 20.11.2012 & 82 & 7. & 294 & 8.8 & -20.5 & & & & 28.0 & 10.0 & 22.0 & 7.3 & & 23.2 & 4.6 & & bdl & & & & & & \\
\hline 26 & 25687 & 09.09 .2016 & 82 & 8 & 359 & 8.8 & -20.5 & -152.4 & & & 32 & 12.0 & 21.0 & 8.2 & 174.4 & 27.0 & bdl & bdl & bdt & 0.41 & 0.19 & & 0.49 & & 0.47 \\
\hline 23 & 25689 & 13.11.2013 & 85 & 8.1 & 158.6 & 8.0 & & & 0.08 & 0.22 & 25.0 & 4.9 & 13.9 & 4.9 & 124.0 & 11.3 & 0.2 & & bdl & 0.15 & 0.10 & 0.04 & 0.32 & 0.00 & 0.33 \\
\hline $24^{8}$ & 25689 & 05.08.2014 & 85 & 7.7 & 297 & 8.3 & -20.6 & -155.1 & & & 28.7 & 11.0 & 19.5 & 7.5 & & 14.0 & bdl & 0.3 & bdl & 0.12 & 0.13 & & 0.47 & & 0.42 \\
\hline \multicolumn{26}{|c|}{ Gdov aquifer $\left(\mathrm{V}_{2 \mathrm{gd}}\right)$} \\
\hline 27 & 122 & 14.09 .2018 & 170 & 7.4 & 1022 & & -19.5 & -145.6 & & & 113.8 & 25.0 & 108.7 & 11.0 & 158.6 & 358.1 & 16.5 & 0.3 & & & & & & 0.56 & \\
\hline $28^{2}$ & 154 & 10.07.1991 & 134 & & & & -20.3 & & & & & & & & & & & & & & & & & & \\
\hline $29^{5}$ & 154 & 12.04 .2005 & 130 & 7.8 & 1649 & 8.5 & -21.0 & & 0.27 & 0.87 & 133.3 & 21.6 & 130.0 & 10.7 & 134.2 & 447.1 & bdl & 0.3 & bdl & & & 0.18 & & 0.48 & \\
\hline $30^{2}$ & 160 & 01.06 .1990 & 142 & & & & -15.0 & & & & & & & & & & & & & & & & & & \\
\hline $31^{1}$ & 160 & 30.07.1991 & 142 & & & & -14.4 & & 0.44 & & & & & & & & & & & & & & & & \\
\hline 32 & 160 & 08.10.2014 & 142 & & 409 & 8.9 & -13.0 & -95.3 & 0.32 & 0.20 & & & & & & 71.0 & & bdl & bdl & 0.49 & & & & & 0.38 \\
\hline $31^{1}$ & 161 & 26.06 .1990 & 140 & 7.7 & & & & & & & 78.0 & 23.6 & 2.4 & 1.0 & 311.2 & 9.2 & 30.5 & & & 0.05 & bdl & 6.10 & & & \\
\hline $34^{3}$ & 161 & 13.04 .2005 & 140 & 7.9 & 788 & 8.8 & -13.6 & & 0. & 0.55 & 88.4 & 13.7 & 54.3 & 10.6 & 231.8 & 139.3 & bdl & 0.8 & bdl & & & 0.10 & & 0.28 & \\
\hline 35 & 170 & 01.04 .2004 & & & & & & & 0.59 & 0.82 & & & & & & & & & & & & & & & \\
\hline $36^{5}$ & 172 & 12.04 .2005 & 136 & 7.9 & 1226 & 8.5 & -20.2 & & 0.48 & 0.72 & 93.8 & 19.3 & 105.0 & 11.4 & 146.4 & 313.0 & bdl & 0.3 & bdl & & & 0.15 & & 0.36 & \\
\hline
\end{tabular}


Table 1. Cont.

\begin{tabular}{|c|c|c|c|c|c|c|c|c|c|c|c|c|c|c|c|c|c|c|c|c|c|c|c|c|c|}
\hline No & $\begin{array}{c}\text { Well } \\
\text { ID }\end{array}$ & Date & $\begin{array}{c}\text { Depth } \\
\text { m }\end{array}$ & $\mathrm{pH}$ & $\begin{array}{c}\text { EC } \\
\mu \mathrm{S} \cdot \mathrm{cm}^{-}\end{array}$ & $\begin{array}{c}\mathrm{T} \\
1{ }^{\circ} \mathrm{C} \\
\end{array}$ & $\begin{array}{l}\delta^{18} \mathrm{O} \\
\% \circ \mathrm{VS}\end{array}$ & $\begin{array}{r}\delta^{2} \mathrm{H} \\
\text { MOW }\end{array}$ & $\begin{array}{c}{ }^{226} \mathrm{Ra} \\
\mathrm{Bq} \cdot \mathrm{I}\end{array}$ & ${ }_{L^{-1}}^{228} \mathrm{Ra}$ & $\mathrm{Ca}^{2+}$ & $\mathrm{Mg}^{2+}$ & $\mathrm{Na}^{+}$ & $\mathrm{K}^{+}$ & $\mathrm{HCO}_{3}{ }^{-}$ & $\mathrm{Cl}^{-}$ & $\mathrm{SO}_{4}{ }^{2-}$ & $\begin{array}{c}\mathrm{Br}^{-} \\
\mathrm{mg} \cdot \mathrm{L}^{-1}\end{array}$ & $\mathrm{Ba}^{2+}$ & $\mathrm{Mn}^{2+}$ & $\mathrm{NH}_{4}{ }^{+}$ & $\mathrm{NO}_{3}{ }^{-}$ & $\mathrm{Sr}^{2+}$ & $\mathrm{Fe}^{2+}$ & $\mathbf{F}^{-}$ \\
\hline \multicolumn{26}{|c|}{ Voronka aquifer $\left(\mathrm{V}_{2 \mathrm{vr}}\right)$} \\
\hline $37^{2}$ & 174 & 14.06 .1988 & 145.4 & 7.7 & & & -12.0 & & 0.24 & & 52.7 & 13.5 & 13.0 & 4.4 & 225.8 & 27.7 & 0.0 & bdl & bdl & & & & & & \\
\hline $38^{5}$ & 183 & 12.04.2005 & 140 & 7.9 & 548 & 9.1 & -21.3 & & 0.34 & 0.46 & 110.6 & 20.4 & 105.0 & 10.7 & 115.9 & 361.3 & bdl & 0.5 & bdl & & & 0.18 & & 0.34 & \\
\hline $39^{5}$ & 188 & 12.04.2005 & 90 & 7.7 & 555 & 8.9 & -21.7 & & 0.17 & 0.32 & 49.7 & 11.4 & 33.0 & 7.3 & 103.7 & 117.0 & 10.3 & 0.3 & bdl & & & 0.09 & & 0.32 & \\
\hline 40 & 188 & 08.10 .2014 & 90 & 8.2 & 513 & 8.7 & -21.6 & -164.9 & 0.28 & 0.29 & 48.7 & 9.8 & 28.2 & 6.7 & 97.6 & 95.9 & 9.0 & bdl & bdl & 0.68 & & & & & 0.48 \\
\hline $41^{1}$ & 296 & 26.10.1989 & 148 & 7.7 & & & & & & & 60.3 & 19.2 & 50.0 & 8.0 & 164.8 & 136.9 & 10.0 & & & 0.10 & 0.46 & bdl & & & \\
\hline 42 & 296 & 09.04.2007 & 148 & 8.0 & & & & & 0.45 & 0.25 & & & & & & & & & & & & & & & \\
\hline 43 & 296 & 14.09.2018 & 148 & 7.5 & 467 & & -14.5 & -105.6 & & & 60.9 & 13.1 & 44.0 & 7.8 & 183.1 & 117.7 & bdl & 0.2 & & & & & & 0.22 & \\
\hline $44^{2}$ & 300 & 14.06.1988 & 165 & 7.6 & & & -14.4 & -105.0 & 0.40 & & 81.0 & 15.9 & 36.7 & 5.2 & 213.6 & 120.9 & 12.7 & bdl & 0.9 & & & & & & \\
\hline 46 & 300 & 16.02.2009 & 165 & 7.6 & & & & & 0.34 & 0.55 & 81.0 & 15.9 & 36.7 & 5.2 & 213.6 & 120.9 & 12.7 & bdl & 0.9 & & & & & & \\
\hline 47 & 300 & 14.09 .2018 & 160 & 7.4 & 496 & & -12.9 & -94.0 & & & 76.6 & 21.4 & 30.0 & 5.4 & 262.4 & 100.7 & bdl & 0.3 & & & & & & 0.37 & \\
\hline 48 & 375 & 05.07.1994 & 160 & 7.8 & & & -22.5 & & & & 85.6 & 20.1 & 108.6 & 11.0 & 189.2 & 280.1 & 5.9 & & & & & & & & \\
\hline $49^{2}$ & 386 & 05.07.1988 & 150 & 7.7 & & & -22.7 & -176.0 & 0.59 & & 104.1 & 22.3 & 78.5 & 10 & 109.8 & 360.5 & 16.9 & bdl & bdl & & & & & & \\
\hline 50 & 412 & 08.10.2014 & 156 & & 349 & 9.2 & -12.2 & -89.4 & 0.25 & 0.09 & & & & & & 74.3 & & bdl & bdl & 0.50 & & & & & 0.35 \\
\hline 51 & 424 & 08.10 .2014 & 150 & 8.3 & 490 & 9.4 & -20.2 & -153.0 & & & 40.6 & 13.5 & 32.1 & 8.5 & 146.4 & 72.1 & 2.2 & bdl & 0.57 & 0.45 & & & & & 0.65 \\
\hline $52^{1}$ & 4460 & 26.10.1989 & 145 & 7.6 & & & & & & & 99.2 & 22.4 & 90.0 & 10.0 & 146.4 & 323.0 & 20.6 & & & 0.25 & 1.20 & bdl & & & \\
\hline $53^{5}$ & 14798 & 13.04.2005 & 159.5 & 7.9 & 526 & 100 & -12.0 & & & & 69.7 & 11.3 & 35.8 & 7.7 & 256.2 & 48.2 & bdl & 0.6 & bdl & & & & & & \\
\hline 54 & 23887 & 09.09 .2016 & 88 & 8.0 & 399 & 8.3 & -20.4 & -153.1 & 0.29 & 0.40 & 120.0 & 19.0 & 110.0 & 11.0 & 130.8 & 360.0 & 17.0 & bdl & bdl & 4.70 & 0.18 & 0.09 & 0.94 & 0.26 & 1.10 \\
\hline 55 & 25686 & 09.09 .2016 & 122 & 8.1 & 1391 & 9.0 & -19.2 & -142.1 & 0.52 & 0.57 & 120.0 & 21.0 & 110.0 & 12.0 & 141.7 & 360.0 & 17.0 & bdl & bdl & 4.60 & 0.19 & & 0.84 & & 1.20 \\
\hline $56^{8}$ & 25688 & 20.11.2012 & 118 & 7.9 & 1003 & 8.9 & -17.7 & & & & 97.0 & 19.0 & 106.0 & 11.0 & & 281.0 & 6.0 & & bdl & & & & & & \\
\hline 57 & 25688 & 09.09.2016 & 118 & 8.0 & 1380 & 9.2 & -17.8 & -130.4 & 0.47 & 0.54 & 120.0 & 20.0 & 110.0 & 12.0 & 174.4 & 350.0 & 17.0 & bdl & bdl & 4.80 & 0.18 & & 0.94 & & 1.20 \\
\hline 58 & 25690 & 13.11.2013 & 122 & 8.2 & 577 & 8.5 & & & 0.69 & 0.44 & 92.0 & 12.1 & 70.4 & 7.9 & 150.0 & 222.1 & 2.4 & & bdl & 2.48 & 0.09 & 0.16 & 0.55 & 0.01 & 0.30 \\
\hline $59^{8}$ & 25690 & 05.08 .2014 & 122 & 7.9 & 1204 & 8.5 & -19.8 & -147.7 & & & 109.4 & 19.2 & 102.6 & 10.7 & & 295.7 & 2.5 & 1.1 & bdl & 2.02 & bdl & & 1.04 & & 0.36 \\
\hline 60 & 25690 & 09.09 .2016 & 122 & 8.1 & 1285 & 9.0 & -19.7 & -146.1 & 0.41 & 0.51 & 110.0 & 19.0 & 100.0 & 11.0 & 130.8 & 330.0 & 16.0 & bdl & bdl & 4.70 & 0.18 & & 0.81 & & bdl \\
\hline $61^{8}$ & 25692 & 08.10 .2014 & 120 & 8. & 1381 & 8.6 & -17.4 & -131.9 & 0.53 & 0.68 & 126.7 & 19.3 & 103.5 & 11.2 & 170.8 & 350.0 & 2.8 & bdl & bdl & 2.18 & & & & & 0.39 \\
\hline 62 & 25692 & 09.09 .2016 & 120 & 8.1 & 1558 & 9.2 & -17.6 & -130.1 & 0.64 & 0.74 & 140.0 & 21.0 & 120.0 & 13.0 & 152.6 & 410.0 & 17.0 & bdl & bdl & 5.70 & 0.21 & & 1.00 & & 1.20 \\
\hline \multicolumn{26}{|c|}{ Voronka + Gdov aquifers } \\
\hline $63^{1}$ & 153 & 26.06 .1990 & & 8.1 & & & & & & & 101.3 & 20.9 & 79.3 & 8.4 & 231.9 & 96.8 & 59.3 & & & 0.05 & bdl & 0.20 & & & \\
\hline $64^{1}$ & 156 & 26.06 .1990 & 110 & 7.4 & & & & & & & 101.6 & 21 & 79.2 & 21.0 & 427.1 & 34.7 & 1.5 & & & 0.03 & bdl & 0.20 & & & \\
\hline 65 & 157 & 09.06 .2014 & 120 & 8.0 & 1274 & 9.3 & -21.2 & -159.9 & 0.59 & 0.60 & 136.6 & 20.5 & 99.5 & 9.9 & & 204.8 & 5.3 & 1.2 & & bdl & 0.87 & & 1.11 & & bdl \\
\hline $66^{1}$ & 162 & 01.08 .1990 & 140 & & & & -16.3 & -111.0 & 0.32 & & & & & & & & & & & & & & & & \\
\hline $67^{5}$ & 162 & 13.04.2005 & 140 & 7.9 & 856 & 8.7 & -17.4 & & 0.52 & 0.16 & 87.4 & 16.0 & 58.6 & 9.0 & 183.0 & 189.3 & bdl & 0.5 & bdl & & & 0.10 & & 0.26 & \\
\hline $68^{1}$ & 163 & 30.07.1991 & 138 & & & & -14.7 & & 0.31 & & 100.1 & 20.3 & 79.7 & & & & & & & & & & & & \\
\hline $69^{2}$ & 163 & 01.06 .1990 & 138 & & & & -15.1 & -102.0 & & & 100.1 & 20.0 & & & & & & & & & & & & & \\
\hline $70^{1}$ & 169 & 26.06 .1990 & & 7.6 & & & & & & & 102.8 & 21.7 & 78.8 & 1.0 & 311.2 & 12.8 & 49.4 & & & 0.03 & bdl & 17.40 & & & \\
\hline $71^{2}$ & 298 & 14.06.1988 & 145 & 7.3 & & & -22.0 & -164.0 & 0.58 & & 103.1 & 21.8 & 78.8 & 9.6 & 134.2 & 293.6 & 10.0 & bdl & 0.7 & & & & & & \\
\hline $72^{1}$ & 299 & 26.10.1989 & 144 & 7.6 & & & & & & & 103.3 & 21.9 & 78.7 & 10.0 & 146.4 & 291.4 & 22.6 & & & 0.20 & 1.80 & bdl & & & \\
\hline $73^{5}$ & 477 & 12.04 .2005 & 90 & 7.7 & 1380 & 9.2 & -22.7 & & 0.65 & 1.18 & 108.8 & 20.4 & 130.0 & 12.9 & 158.6 & 350.6 & 26.5 & bdl & bdl & & & 0.14 & & 0.62 & \\
\hline $74^{1}$ & 485 & 26.10 .1989 & 145 & 8 & & & & & & & 104.3 & 22.4 & 78.4 & 4.0 & 238.0 & 39.0 & 0.0 & & & 0.22 & 0.56 & bdl & & & \\
\hline 75 & 485 & 14.09 .2018 & 145 & 7.6 & 285 & & -11.7 & -84.1 & & & 50.1 & 12.5 & 14.2 & 4.6 & 250.2 & 8.5 & bdl & 0.4 & & & & & & 0.20 & \\
\hline $76^{2}$ & 115 & 07.07.1991 & 120 & 8.3 & 200 & & -21.9 & & & & 159.5 & 15.3 & 116.0 & 8.0 & 73.2 & 453.4 & 21.4 & & & 0.08 & & & & & \\
\hline $77^{3}$ & 1152 & 05.07.1994 & 122 & 7.9 & & & -21.4 & & & & 150.7 & 14.7 & 122.0 & 8.3 & 85.4 & 455.6 & 18.9 & & & & & & & & \\
\hline $78^{4}$ & 1152 & 28.09.1995 & 122 & & & & -21.3 & & & & & & & & & & & & & & & & & & \\
\hline
\end{tabular}


Table 1. Cont.

\begin{tabular}{|c|c|c|c|c|c|c|c|c|c|c|c|c|c|c|c|c|c|c|c|c|c|c|c|c|c|}
\hline No & $\begin{array}{c}\text { Well } \\
\text { ID }\end{array}$ & Date & $\begin{array}{c}\text { Depth } \\
\text { m }\end{array}$ & $\mathrm{pH}$ & $\begin{array}{c}\text { EC } \\
\mu \mathrm{S} \cdot \mathrm{cm}^{-}\end{array}$ & $\begin{array}{r}\mathrm{T} \\
1{ }^{\circ} \mathrm{C} \\
\end{array}$ & $\begin{array}{l}\mathcal{\delta}^{18} \mathrm{O} \\
\%_{0} \text { VSN }\end{array}$ & $\begin{array}{r}\delta^{2} \mathrm{H} \\
\text { MOW }\end{array}$ & $\begin{array}{r}{ }^{226} \mathrm{Ra} \\
\mathrm{Bq} \cdot \mathrm{I}\end{array}$ & ${ }_{L^{-1}}^{228} \mathrm{Ra}$ & $\mathrm{Ca}^{2+}$ & $\mathrm{Mg}^{2+}$ & $\mathrm{Na}^{+}$ & $\mathrm{K}^{+}$ & $\mathrm{HCO}_{3}{ }^{-}$ & $\mathrm{Cl}^{-}$ & $\mathrm{SO}_{4}{ }^{2-}$ & $\begin{array}{c}\mathrm{Br}^{-} \\
\mathrm{mg} \cdot \mathrm{L}^{-1}\end{array}$ & $\mathrm{Ba}^{2+}$ & $\mathrm{Mn}^{2+}$ & $\mathrm{NH}_{4}{ }^{+}$ & $\mathrm{NO}_{3}{ }^{-}$ & $\mathrm{Sr}^{2+}$ & $\mathrm{Fe}^{2+}$ & $\mathrm{F}^{-}$ \\
\hline \multicolumn{26}{|c|}{ Voronka aquifer $\left(\mathrm{V}_{2 \mathrm{vr}}\right)$} \\
\hline 79 & 1152 & 28.10 .2014 & 122 & & & & -21.5 & & & & 146.9 & 13.1 & 150.0 & 7.3 & 85.4 & 482.2 & 7.0 & & & & & & & & \\
\hline $80^{4}$ & 4734 & 12.08.1996 & 100 & & & & -22.0 & -161. & & & 63.1 & 21.3 & 43.4 & 7.3 & 146.4 & 185.8 & 6.6 & & & & & & & & \\
\hline $81^{7}$ & 4734 & 27.03.2007 & 100 & 8.0 & 728 & 9.3 & -22.0 & & & & & & & & & & & & & & & & & & \\
\hline 82 & 4734 & 02.09 .2016 & 100 & 7.9 & 1069 & 9.4 & -22.5 & -168.4 & & & 82.0 & 17.0 & 95.0 & 11.0 & 152.6 & 240.0 & 19.0 & bdl & bdl & 3.40 & 0.19 & & 0.55 & & 1.00 \\
\hline $83^{5}$ & 5157 & 12.04 .2005 & 125 & 7.8 & 445 & 9.1 & -12.5 & & 0.43 & 0.23 & 52.5 & 15.9 & 20.0 & 9.3 & 280.7 & 22.3 & bdl & 0.3 & bdl & & & 0.08 & & 0.29 & \\
\hline 84 & 11569 & 01.07.2016 & 100 & 8.0 & 409 & 8.5 & -21.5 & -161.7 & & & 61.0 & 9.8 & 35.0 & 6.3 & & 150.0 & 15.0 & 0.6 & 3.3 & 1.40 & bdl & & 0.24 & & 0.34 \\
\hline 85 & 15831 & 13.11.2013 & 123.3 & 8.3 & 527 & 8.2 & -22.6 & -169.7 & 0.77 & 0.52 & 71.8 & 10.7 & 63.3 & 7.5 & 149.5 & 176.9 & 17.2 & & bdl & 0.75 & 0.18 & 0.09 & 0.46 & 0.01 & 0.28 \\
\hline 86 & 15841 & 14.04 .2005 & 117 & 7.9 & 933 & 9.0 & -22.7 & & 0.55 & 0.67 & 64.7 & 16.5 & 82.7 & 10.0 & 170.8 & 199.6 & bdl & 0.2 & bdl & & & 0.08 & & 0.25 & \\
\hline 87 & 16557 & 09.06 .2014 & 110 & 8.1 & 393 & 9.2 & -21.6 & -163.4 & 0.12 & 0.10 & 45.1 & 8.6 & 24.6 & 6.7 & & 59.8 & 10.2 & bdl & bdl & bdl & 0.08 & & 0.36 & & 0.54 \\
\hline 88 & 16557 & 28.10.2014 & 110 & & & & -21.6 & -162.2 & & & & & & & & & & & & & & & & & \\
\hline 89 & 485 & 14.09.2018 & 145 & 7.6 & 285 & & -11.7 & -84.1 & 0.18 & 0.30 & 50.1 & 12.5 & 14.2 & 4.6 & 250.2 & 8.5 & bdl & 0.4 & & & & & & 0.20 & \\
\hline \multicolumn{26}{|c|}{ Crystalline basement aquifer } \\
\hline $90^{2}$ & 1151 & 06.09 .1994 & 160 & 7.7 & & & -21.2 & & & & 206.0 & 32.6 & 211.0 & 12.5 & 79.3 & 735.4 & 27.6 & & & & & & & & \\
\hline $91^{5}$ & 1151 & 25.04 .2001 & 160 & 8.5 & 1540 & 8.8 & -21.3 & & & & 188.0 & 25.2 & 210.0 & 11.7 & 64.1 & 629.0 & 14.8 & & & & & & & & \\
\hline
\end{tabular}

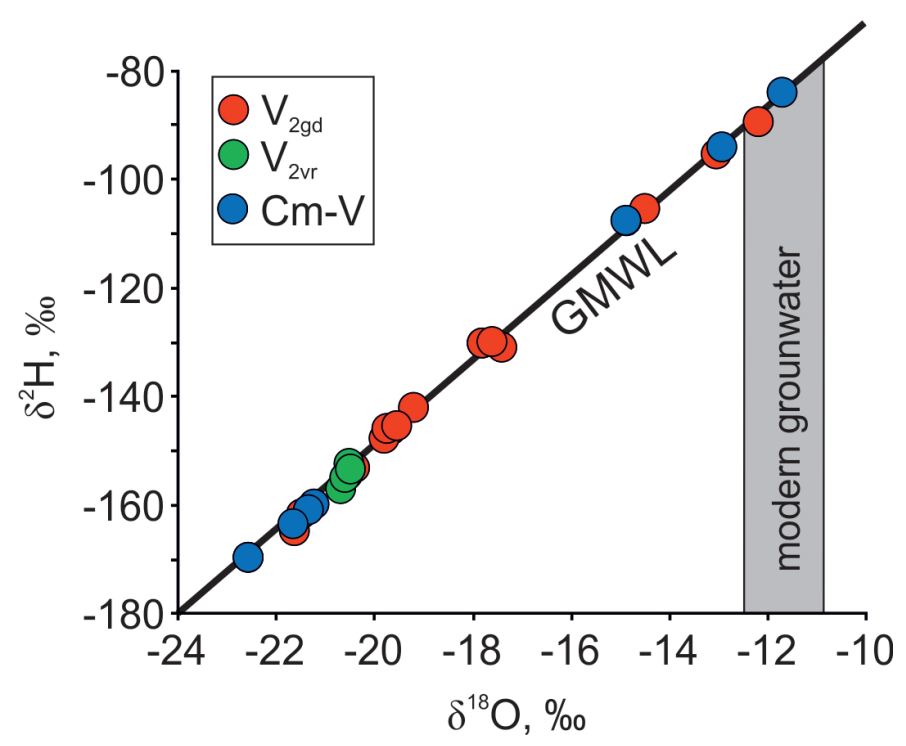

Figure 2. $\delta^{18} \mathrm{O}$ and $\delta^{2} \mathrm{H}$ isotopic compositions of groundwater in the $\mathrm{Cm}-\mathrm{V}$ aquifer system in the Viimsi peninsula. Shallow groundwater data are from Raidla et al. [19]. 
The changes of groundwater quality (Figure 3) in the past have been most drastic in the deeper Gdov aquifer (Figure 4). However, the changes in the isotopic composition of groundwater resulting from the changes in groundwater abstraction on the peninsula have not been similar. The spatial distribution of $\delta^{18} \mathrm{O}$ values from the period 1987 to 1992 (Figure 4a), when water consumption was at its maximum, is similar to the situation observed in the period from 2005 to 2009 (Figure 4c). In those periods groundwater with an isotopic composition markedly different from the baseline values of $\mathrm{Cm}-\mathrm{V}$ aquifer system $\left(\delta^{18} \mathrm{O}\right.$ values from $-18.5 \%$ to $\left.-23 \%\right)$ are only observed on the south-western part of the peninsula near the deep buried valley. The spatial distribution of $\delta^{18} \mathrm{O}$ values in the period from 2013 to 2016 shows that the area, where the isotopic composition of groundwater has drastically changed, has grown much wider towards the central part of the peninsula, where the main groundwater intake constructed in 2012 is situated (Figure 4a-c). The main trend observed in the isotopic composition of groundwater is the increase of $\delta^{18} \mathrm{O}$ values in wells located closer to the buried valley in the south-western part of the peninsula (Figure 4a). Thus, the changes in the isotopic composition of groundwater could primarily be related to the recharge of modern meteoric water into the $\mathrm{Cm}-\mathrm{V}$ aquifer system from the Quaternary aquifer in the buried valley. This, in turn, would lead to mixing between the intruding modern groundwater and glacial paleo-groundwater in the aquifer system.

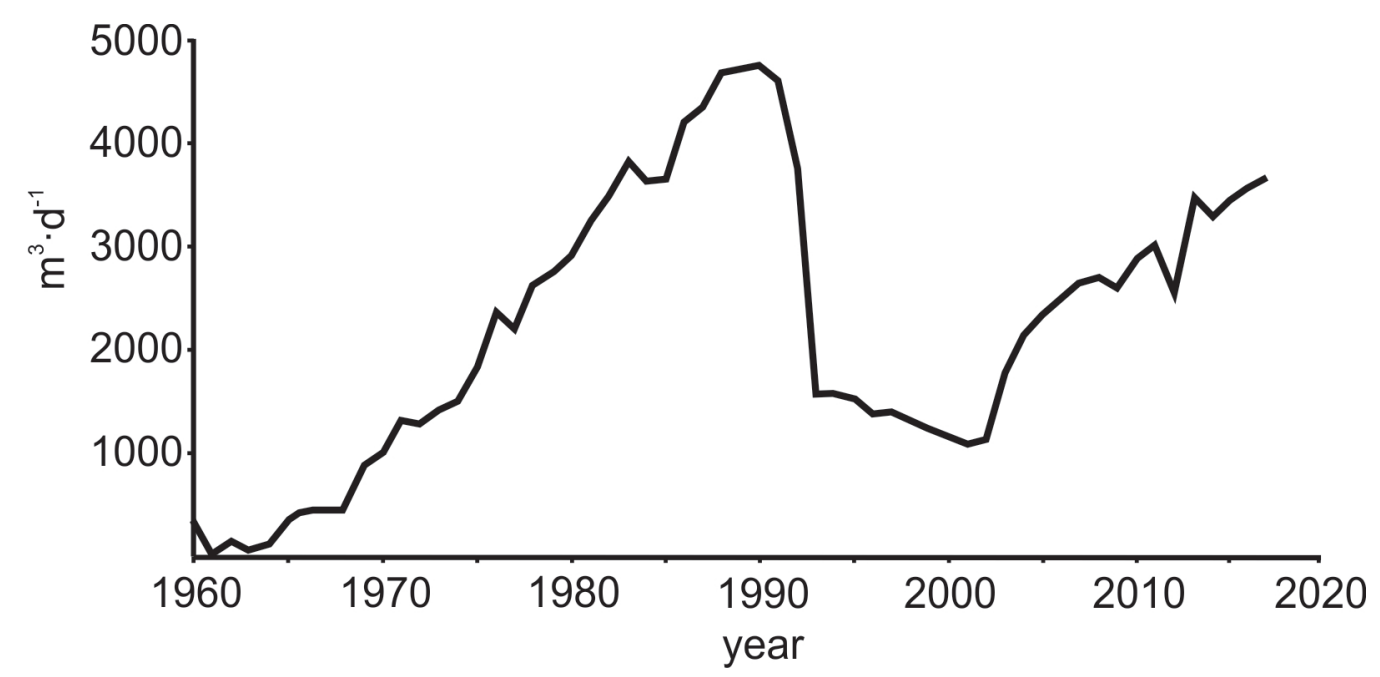

Figure 3. Groundwater abstraction in the Viimsi peninsula from 1960 to the present.

In 1987 to 1992, a significant increase in $\mathrm{HCO}_{3}{ }^{-}$and $\mathrm{SO}_{4}{ }^{2-}$ concentrations is observed together with enrichment in isotopic composition (Table 1; Figure 4a). However, in recent years (from 2013 to 2016), changes in isotopic composition are mainly accompanied by increased concentration of $\mathrm{Cl}^{-}$ (Table 1; Figure $4 \mathrm{~b}, \mathrm{~d}, \mathrm{f}$ ). The increase in $\mathrm{Cl}^{-}$concentrations is accompanied by an increase in $\mathrm{Na}^{+}$and $\mathrm{Ca}^{2+}$ concentrations and the decrease in $\mathrm{HCO}_{3}{ }^{-}$concentrations (Figure 5).

Conservative geochemical tracers, such as $\delta^{18} \mathrm{O}$ and $\mathrm{Cl}^{-}$indicate the mixing trend between three components (Figure 6), which is somewhat similar to the three-component mixing model proposed by Raidla et al. [18] for the Cm-V aquifer system on a regional scale. According to this model, the mixing in the $\mathrm{Cm}-\mathrm{V}$ aquifer system has occurred between water originating from modern recharge, glacial meltwater that infiltrated in the Pleistocene and saline formation water. The mixing between fresh meteoric water and groundwater of glacial origin is clearly detectable, but the water with strongly depleted $\delta^{18} \mathrm{O}$ values has a wide variability in $\mathrm{Cl}^{-}$concentrations and the exact origin of this endmember is not clear at present. Suursoo et al. [24] proposed two sources for $\mathrm{Cl}^{-}$: The intrusion of seawater, or inflow of saline water from the underlying crystalline basement. 

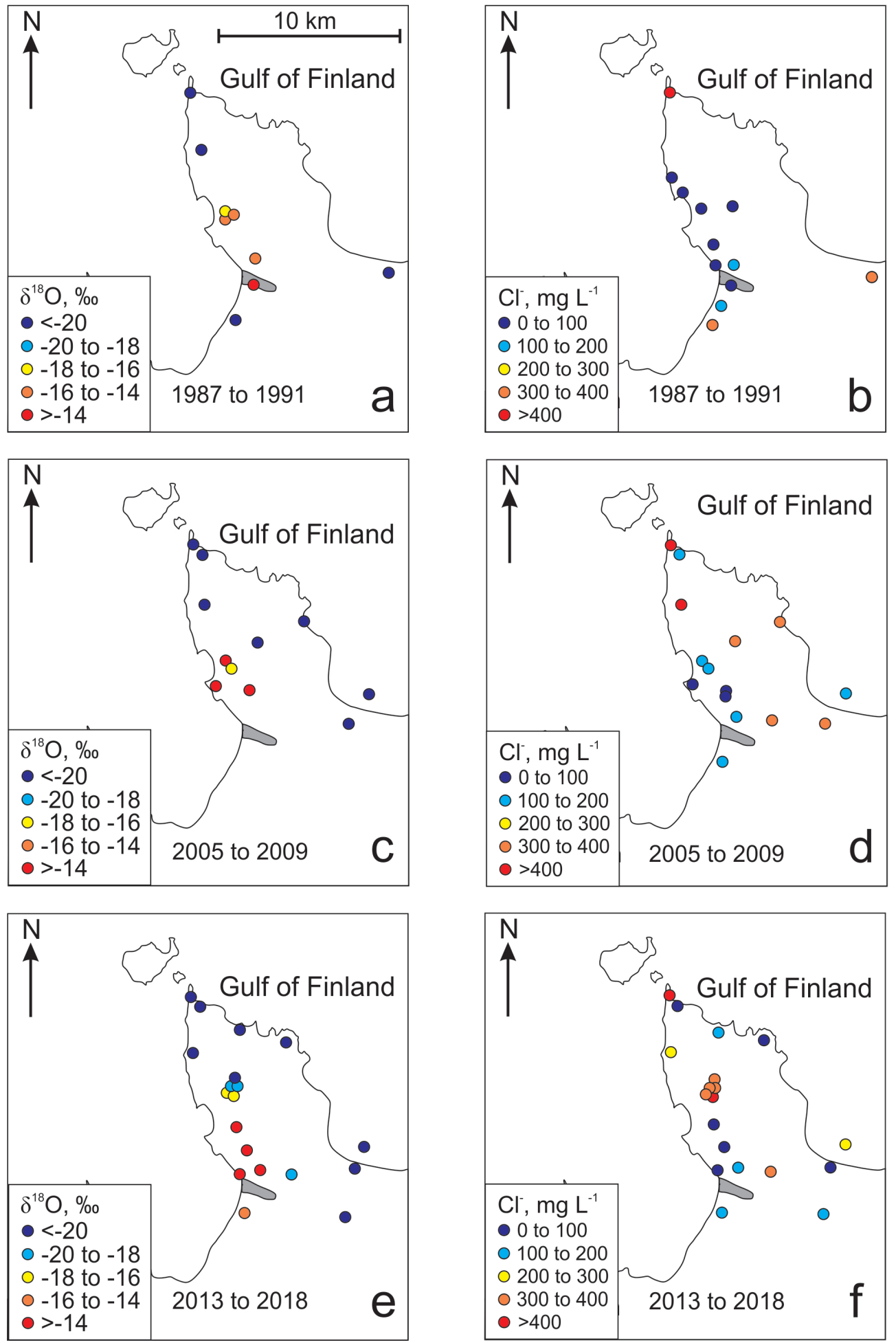

Figure 4. Variations of $\delta^{18} \mathrm{O}$ and $\mathrm{Cl}^{-}$in the Gdov aquifer in the Viimsi peninsula in different periods of time. 


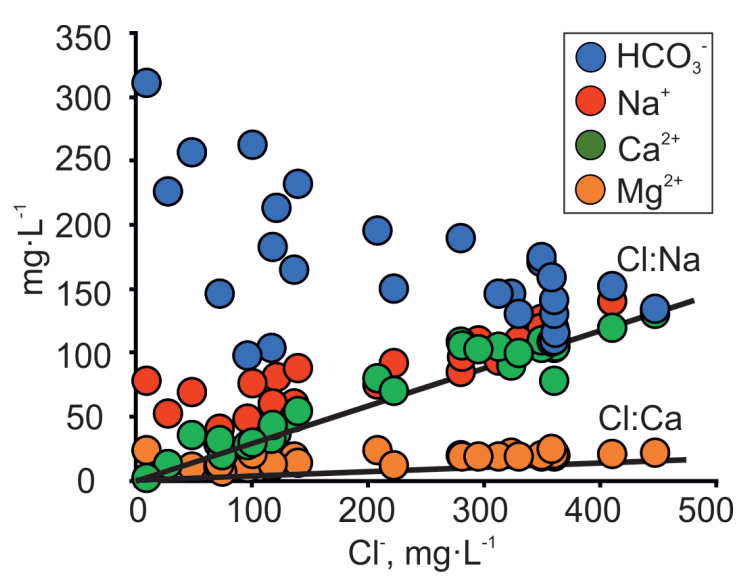

Figure 5. Co-variance between major ions and $\mathrm{Cl}^{-}$concentration in the $\mathrm{Cm}-\mathrm{V}$ groundwater of the Viimsi peninsula. Lines $\mathrm{Cl}: \mathrm{Na}$ and $\mathrm{Cl}: \mathrm{Ca}$ on the figure denote seawater dilution lines.

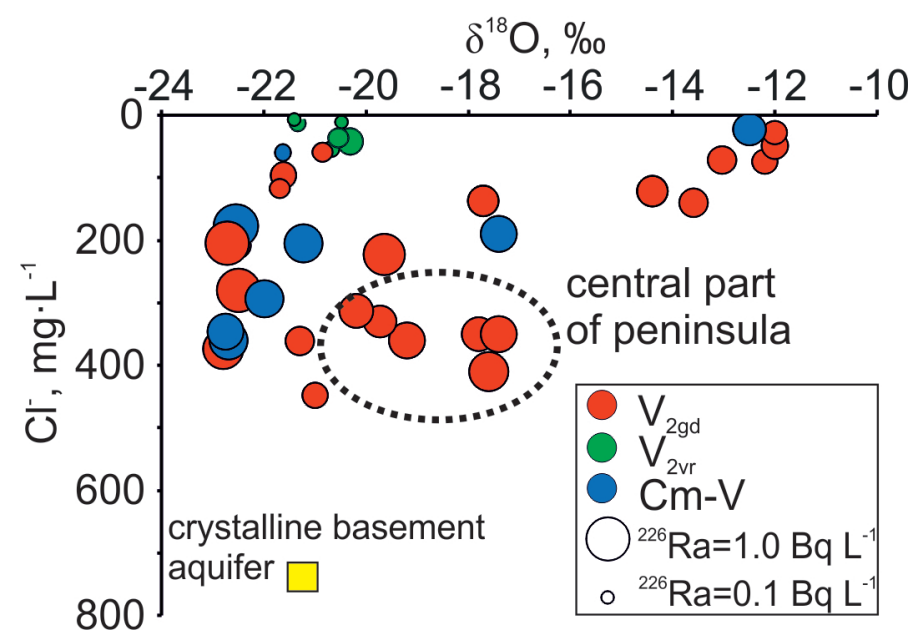

Figure 6. Co-variance between $\delta^{18} \mathrm{O}$ and $\mathrm{Cl}^{-}$relation with ${ }^{226} \mathrm{Ra}$ variations in $\mathrm{Cm}-\mathrm{V}$ groundwaters of the Viimsi peninsula.

The rocks forming the Gdov aquifer outcrop under the Baltic Sea and the $\mathrm{Na} / \mathrm{Cl}$ ratios of groundwater follow the seawater dilution/evaporation line (Figure 5). This makes seawater intrusion a plausible source of salinity. However, since similar $\mathrm{Na} / \mathrm{Cl}$ ratios have been detected in palaeo-groundwater all over the $\mathrm{Cm}-\mathrm{V}$ aquifer system [18], this is not a definite indicator of seawater intrusion in the area. Several lines of evidence suggest that the seawater intrusion cannot explain the changes observed in the chemical and isotopic composition of groundwater in the study area.

Firstly, the location of salinisation and freshening zones in the study area are uncharacteristic to seawater intrusion. Freshwater with heavier isotopic composition is found on the south-western coast of the peninsula, where the buried valley is located, but the recent rise in $\mathrm{Cl}^{-}$concentrations is observed in the central part of the peninsula, and not on the coast (Figure $4 \mathrm{f}$ ).

Secondly, the rising salinity is accompanied by an increase in $\mathrm{Ca}^{2+}$ concentrations, but the latter are low in seawater, which is dominated by $\mathrm{Na}^{+}$and $\mathrm{Mg}^{2+}$ [49]. The covariation between $\mathrm{Cl}^{-}$and $\mathrm{Ca}^{2+}$ values in groundwater on the Viimsi peninsula (Figure 5) suggests that the salinity increase is related to the underlying crystalline basement as the groundwater plots away from the seawater dilution line (SDL) of the Baltic Sea [50]. A similar trend in the $\mathrm{Cl}^{-}$and $\mathrm{Ca}^{2+}$ relation has been observed in the Kopli peninsula (Figure 1a), where isotopic signatures of $\delta^{18} \mathrm{O}$ and ${ }^{14} \mathrm{C}$ revealed the increase in salinity by palaeogroundwater of glacial origin, which had intruded from the underlying crystalline basement [42]. 
It can be proposed that the three-component mixing observed in the Gdov aquifer has occurred in two stages. In natural conditions, the piezometric levels of the $\mathrm{Cm}-\mathrm{V}$ aquifer system in the Viimsi area were above seawater level [51] and the aquifer contained glacial palaeogroundwater (Figure 7a). The groundwater abstraction that started in the 1960s (Figure 3) lowered the hydraulic head by several metres, the strongest impact occurring first (in 1960 to 1992) close to the buried valley, where infiltration of modern meteoric water had become the main compensation mechanism for the lowered hydraulic heads (Figure $7 \mathrm{~b}$ ). The influx of groundwater containing $\mathrm{O}_{2}$ and having higher $\mathrm{CO}_{2}$ partial pressure resulted in atypically high $\mathrm{HCO}_{3}{ }^{-}, \mathrm{SO}_{4}{ }^{2-}$ and $\mathrm{NO}_{3}{ }^{-}$concentrations in the $\mathrm{Cm}-\mathrm{V}$ groundwater in the period from 1987 to 1991 (Table 1). In 2012, the new groundwater intake started to operate in the central part of the peninsula and the main groundwater abstraction shifted to this area (Figure 7c). The buried valley lay too far for the abstraction to be directly compensated by infiltration of modern groundwater. Thus, the upwelling of saline groundwater from the underlying crystalline basement became the dominating hydraulic head compensation mechanism, which was accompanied by an abrupt rise in $\mathrm{Cl}^{-}$and $\mathrm{Ca}^{2+}$ concentrations in groundwater. The deterioration of groundwater quality in the newly founded intake led to reopening of wells in the southern part of the peninsula. This new situation has decreased the inflow from the underlying crystalline basement aquifer and has supported the flow of modern groundwater from the buried valley to the more central parts of the peninsula (Figure 7d). Chemical and isotopic compositions of groundwater suggest that at present, the front of modern groundwater has almost reached the new groundwater intake and the wells receive groundwater from both the crystalline basement and the buried valley. As a result, $\mathrm{Cl}^{-}$concentrations have stabilised or even decreased a little and the water's stable oxygen isotope composition has become enriched with respect to glacial palaeogroundwater which occupied the aquifer system in natural conditions. According to this reasoning, the mixing of three different water types (glacial palaeogroundwater, brackish/saline groundwater from the crystalline basement aquifer and fresh modern groundwater from the buried valley) induced by groundwater abstraction explains the changes in groundwater quality and isotopic composition observed on the Viimsi peninsula (Figure 7d). 

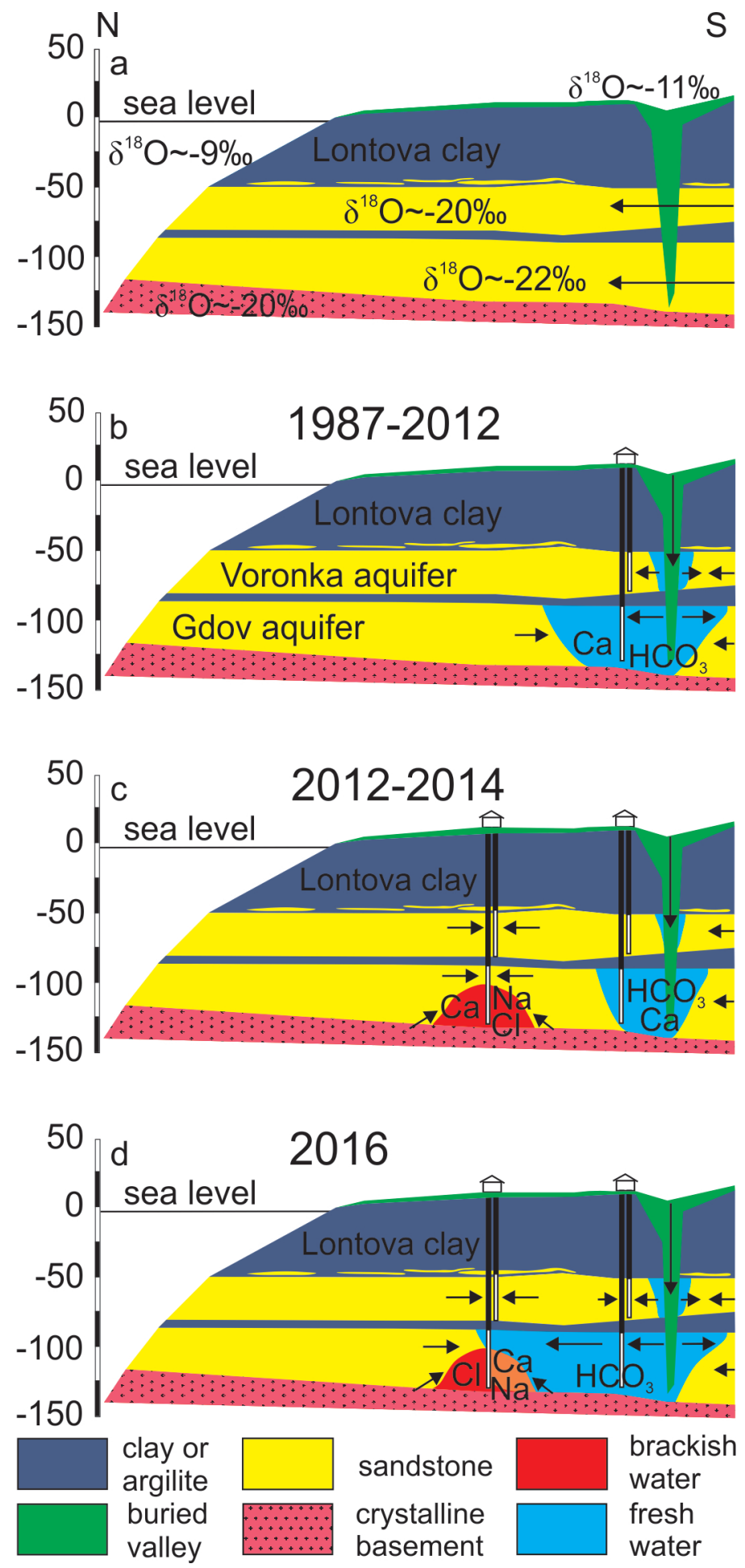

Figure 7. A conceptual model for the formation of chemical composition of groundwater in the $\mathrm{Cm}-\mathrm{V}$ aquifer system of the Viimsi peninsula.

\subsection{Spatial Distribution and the Origin of Radium in Groundwater}

One of the important issues that has occurred with increased abstraction and subsequent changes in the chemical composition of the groundwater in the Gdov aquifer is the rise in radium activity [24]. Activities of ${ }^{226} \mathrm{Ra}$ and ${ }^{228} \mathrm{Ra}$ in the Gdov aquifer of the Viimsi peninsula vary from 0.1 to $2.14 \mathrm{~Bq} \cdot \mathrm{L}^{-1}$ and from 0.1 to $1.12 \mathrm{~Bq} \cdot \mathrm{L}^{-1}$, respectively. The rise in $\mathrm{Cl}^{-}$concentrations on the peninsula are caused 
by the inflow of water from the crystalline basement, as shown in the previous section. This water is brackish or saline, and typically has a higher content of radionuclides [25], because of $U$ and Th present in the crystalline basement [52,53]. The gamma radiation well-logs also support this understanding (Appendix A). Our results show the covariance between $\mathrm{Cl}^{-}$and $\mathrm{Ra}$, although the correlation with $\mathrm{Cl}^{-}$is better for ${ }^{228} \mathrm{Ra}$ than for ${ }^{226} \mathrm{Ra}$ (Figure 8). This observation indicates that the source of $\mathrm{Cl}^{-}$ must lie close to the sampling wells, due to relatively short half-life of ${ }^{228} \mathrm{Ra}$ (5.75 years). By contrast, ${ }^{226} \mathrm{Ra}$ (half-life is 1,600 years) has a wider spread from the mixing line of $\mathrm{Ra}$ and $\mathrm{Cl}^{-}$. This could indicate that the ${ }^{226} \mathrm{Ra}$ origins from several sources or it has been affected by substantial geochemical changes, due to its longer residence time in groundwater.

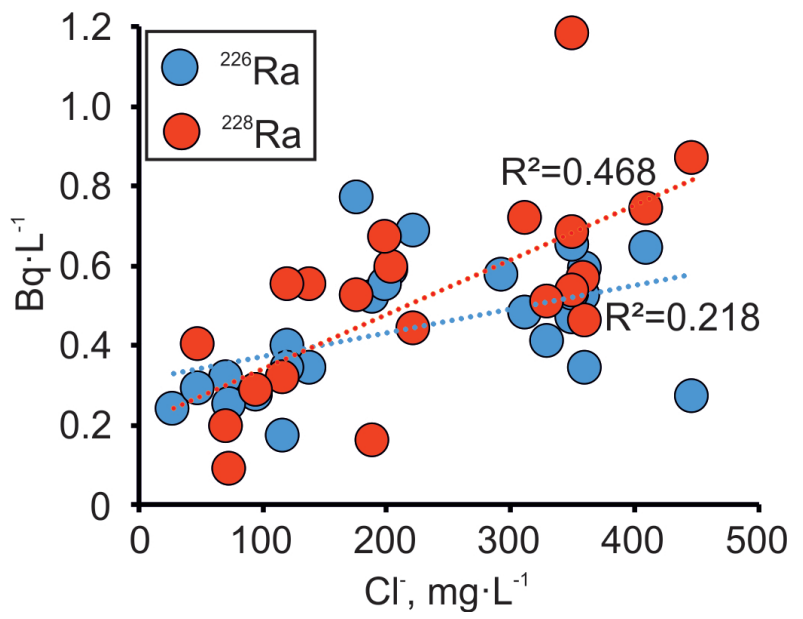

Figure 8. Variations in the activity of Ra isotopes in dependence on $\mathrm{Cl}^{-}$concentration in the $\mathrm{Cm}-\mathrm{V}$ groundwater in the Viimsi peninsula.

The clay sediments having the ability to adsorb cations [54] are more abundant in the deeper parts of the Gdov aquifer in the contact-surface between Ediacaran sandstones and the crystalline basement [54-56]. The increase in $\mathrm{Cl}^{-}$concentrations in the Gdov aquifer has brought about the increase in $\mathrm{Ca}^{2+}$ and $\mathrm{Na}^{+}$concentrations (Figure 5) which can induce cation exchange [15]. In cation exchange processes, Ra behaves similarly to other divalent cations and can take part in absorption/desorption processes [57]. However, a clear inverse relationship between $\mathrm{Ca}^{2+}$ and $\mathrm{Na}^{+}$, which is indicative of absorption processes [49], is not detectable in the Gdov aquifer. Moreover, Swarzenski et al. [58] have shown that other bivalent cations $\left(\mathrm{Ca}^{2+}, \mathrm{Mg}^{2+}\right)$ clearly prevail over $\mathrm{Ra}^{2+}$ in weakly cemented quartzose sands like Ediacaran sandstones, because the amount of rock surface where the cations could possibly be adsorbed, is smaller [30].

The second source of ${ }^{226} \mathrm{Ra}$ could be a dissolution of carbonate cement from the aquifer matrix. ${ }^{226} \mathrm{Ra}$ has a clear positive correlation with both the saturation index of calcite $(\mathrm{R}=0.669)$ and the saturation index of disordered dolomite $(\mathrm{R}=0.640)$. At the same time, ${ }^{228} \mathrm{Ra}$ correlation with saturation indexes remains lower, 0.483 and 0.417, respectively. Chemical data from 1988 and 1991 shows anomalously high $\mathrm{Ca}^{2+}$ concentrations accompanied by increase in $\mathrm{Mg}^{2+}$ and $\mathrm{HCO}_{3}{ }^{-}$concentrations that were not observed in 2013 to 2016 (Table 1). Additionally, concentrations of $\mathrm{SO}_{4}{ }^{2-}$ increased and $\mathrm{NH}_{4}{ }^{+}$decreased when $\mathrm{NO}_{3}{ }^{-}$appeared in groundwater. We interpret such changes as oxidation of pyrite and $\mathrm{NH}_{4}{ }^{+}$by aerobic groundwater, in parallel with $\mathrm{pH}$ buffering through the dissolution of carbonates. Disordered dolomite is probably the free proton neutraliser, because it is the dominant carbonate mineral in the cement of Ediacaran sandstones in the study area [54], and its saturation indexes in Gdov groundwater are systematically below zero (Table 2). On the other hand, Table 2 shows that Gdov groundwater is mostly oversaturated with respect to calcite. Mixing between bicarbonate-rich modern groundwater and Ca-rich crystalline water might have produced calcite precipitation, due to emerged chemical disequilibrium [49], which in turn could have influenced the activity concentrations 
of $\mathrm{Ra}$ as the $\mathrm{Ca}^{2+}$ chemical analogue [58]. However, the Ra dissolution/precipitation in the peninsula needs more detailed research in the future.

Table 2. Saturation indexes (SI) of the $\mathrm{Cm}-\mathrm{V}$ groundwaters in the Viimsi peninsula.

\begin{tabular}{cccccc}
\hline No & Well ID & SI-calcite & SI-dolomte & SI-dolomite (disordered) & SI-CO $_{\mathbf{2}}(\mathbf{g})$ \\
\hline 2 & 124 & 0.05 & -0.32 & -0.93 & -2.94 \\
5 & 171 & 0.41 & 0.43 & -0.18 & -3.07 \\
6 & 179 & -0.11 & -0.45 & -1.07 & -2.65 \\
9 & 187 & -0.30 & -0.90 & -1.51 & -2.72 \\
10 & 187 & -0.09 & -0.82 & -1.44 & -2.90 \\
15 & 1153 & -0.29 & -1.00 & -1.61 & -2.76 \\
18 & 8914 & 0.17 & 0.04 & -0.58 & -2.97 \\
21 & 19570 & 0.37 & 0.21 & -0.40 & -2.82 \\
23 & 25689 & -0.03 & -0.64 & -1.25 & -3.10 \\
29 & 154 & 0.28 & -0.12 & -0.73 & -2.81 \\
34 & 161 & 0.49 & 0.30 & -0.32 & -2.66 \\
36 & 172 & 0.29 & 0.02 & -0.59 & -2.86 \\
38 & 183 & 0.25 & -0.10 & -0.72 & -2.97 \\
39 & 188 & -0.27 & -1.06 & -1.68 & -2.80 \\
40 & 188 & 0.21 & -0.15 & -0.77 & -3.32 \\
46 & 300 & 0.11 & -0.36 & -0.97 & -2.61 \\
53 & 14798 & 0.46 & 0.24 & -0.37 & -3.08 \\
55 & 25686 & 0.57 & 0.50 & -0.11 & -2.89 \\
57 & 25688 & 0.55 & 0.45 & -0.16 & -3.14 \\
58 & 25690 & 0.62 & 0.48 & -0.14 & -3.11 \\
60 & 25690 & 0.50 & 0.37 & -0.24 & -3.20 \\
61 & 25692 & 0.87 & 1.05 & 0.43 & -3.05 \\
62 & 25692 & 0.65 & 0.60 & -0.01 & -2.76 \\
67 & 162 & 0.38 & 0.15 & -0.47 & -2.64 \\
73 & 477 & 0.16 & -0.28 & -0.89 & -2.47 \\
83 & 5157 & 0.28 & 0.16 & -0.45 & -2.24 \\
85 & 15831 & 0.62 & 0.53 & -0.08 & -2.32 \\
86 & 15841 & 0.23 & -0.02 & -0.63 & -1.06 \\
89 & 485 & 0.02 & -0.45 & &
\end{tabular}

\subsection{Secondary U Deposits as the Source of ${ }^{226} R a$}

Although higher Ra activities observed in the $\mathrm{Cm}-\mathrm{V}$ aquifer system have been attributed to the prolonged contact with the rocks of the crystalline basement [26,43], Ra is also present in the Voronka aquifer $\left({ }^{226} \mathrm{Ra}\right.$ from values under the detection limit to $0.4 \mathrm{~Bq} \cdot \mathrm{L}^{-1} ;{ }^{228} \mathrm{R}$ from 0.1 to $0.3 \mathrm{~Bq} \cdot \mathrm{L}^{-1}$ ), which has no direct contact with the crystalline basement. Concentrations of $\mathrm{Cl}^{-}$and $\delta^{18} \mathrm{O}$ values in the Gdov aquifer have a clear mixing trend with modern groundwater, but the ${ }^{226} \mathrm{Ra}$ values in groundwater having modern isotopic signature stay moderately high (Figure 6) and ${ }^{226} \mathrm{Ra}$ activity percentage in total radium activity increases towards the buried valley (Figure 9). This may indicate heterogeneous spreading of $U$ in surfaces of sedimentary rocks, in which case locally high $U$ contents could occur resulting in higher Ra activities [59].

The radium isotopes often behave in a similar manner during chemical processes. Contents of ${ }^{226} \mathrm{Ra}$ and ${ }^{228} \mathrm{Ra}$ in water tend to depend on the presence of their parent nuclides $\left({ }^{238} \mathrm{U}\right.$ and ${ }^{232} \mathrm{Th}$ respectively) in the surrounding porous media [60,61]. Uranium and thorium are not fractionated significantly from each other during partial melting and subsequent differentiation in magma [62]; thus, their variations in magmatic rock, like the one encountered in the Estonian crystalline basement, should be similar. However, in fluids they behave quite differently. The ${ }^{228}$ Ra parent mineral Th stays immobile in water, whereas the ${ }^{226}$ Ra parent mineral $U$ can be very mobile. Uranium concentrations in groundwater are strongly controlled by redox conditions, because $U$ can be present as $\mathrm{U}^{4+}$ ion with very low solubility in anoxic conditions and as highly soluble and mobile $\mathrm{U}^{6+}$ ion in oxidizing environments [36,63-65]. These chemical differences can produce variations in $U$ and Th contents in the aquifer matrix (in rock) and through this, variations of Ra isotope activity in water. 
Anoxic conditions predominate in the $\mathrm{Cm}-\mathrm{V}$ aquifer system, but Raidla et al. [21,48] show that oxygen-rich conditions dominated in the aquifer system in the relatively short infiltration period during the advance and maximum extent of the Scandinavian ice sheet in the Last Glacial Maximum. Vaikmäe et al. [20] have shown that groundwater in the $\mathrm{Cm}-\mathrm{V}$ aquifer system in North-Estonia is characterised by enormous amounts of excess air (oversaturation of $\mathrm{Ne}$ up to $200-400 \%)$. The anomalous amount of excess air in groundwater has been thought to originate from air bubbles found in glacial basal ice [20]. Alternatively, Kipfer et al. [66] and Grundl et al. [67] have explained that the high Ne excess air amounts observed in groundwater of glacial origin are derived from trapped air pockets in englacial drainage systems, which behave in the same manner as trapped bubbles in porous sediments. The relation between $\mathrm{Ar}$ and $\mathrm{Kr}$ in $\mathrm{Cm}-\mathrm{V}$ groundwater from the Viimsi peninsula follows the trend expected for the addition of air to air-equilibrated water (Figure 10; Table 3). These results agree with both hypotheses, because noble gas composition in ice generally corresponds to atmospheric composition, except for $\mathrm{He}$ and $\mathrm{Ne}$, which can diffuse out of the firn during the firnification process, due to its smaller atomic radius [68]. As the concentrations of noble gases, such as $\mathrm{Ar}$ and $\mathrm{Kr}$ in subglacial meltwater correspond to atmospheric composition, we propose that a similar relationship can also be applied for other atmospheric gases, like $\mathrm{O}_{2}$. For instance, by knowing the concentration of Ar in groundwater, one can also calculate the initial oxygen concentration in glacial meltwater. Figure 10 indicates that the glacial meltwater, which intruded the aquifer system in the Pleistocene, was 1.5 to 2.5 times oversaturated with respect to $\mathrm{O}_{2}$.

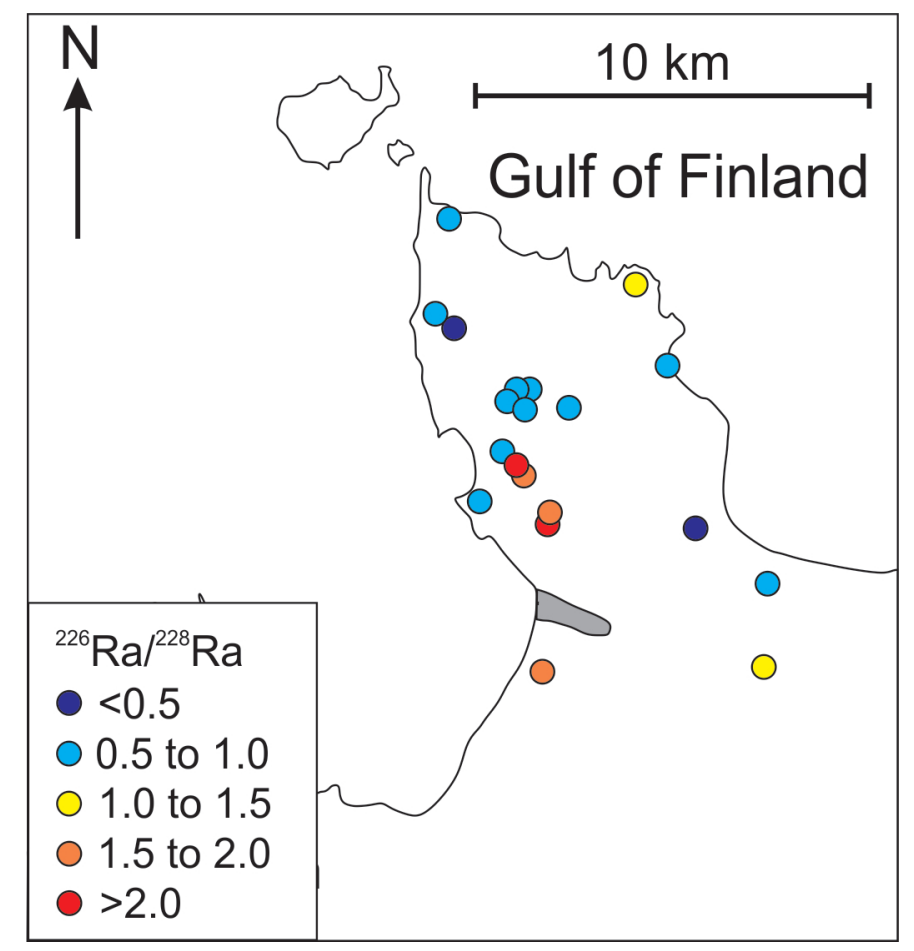

Figure 9. Variations of Ra isotopes in $\mathrm{Cm}-\mathrm{V}$ groundwater of the Viimsi peninsula. 

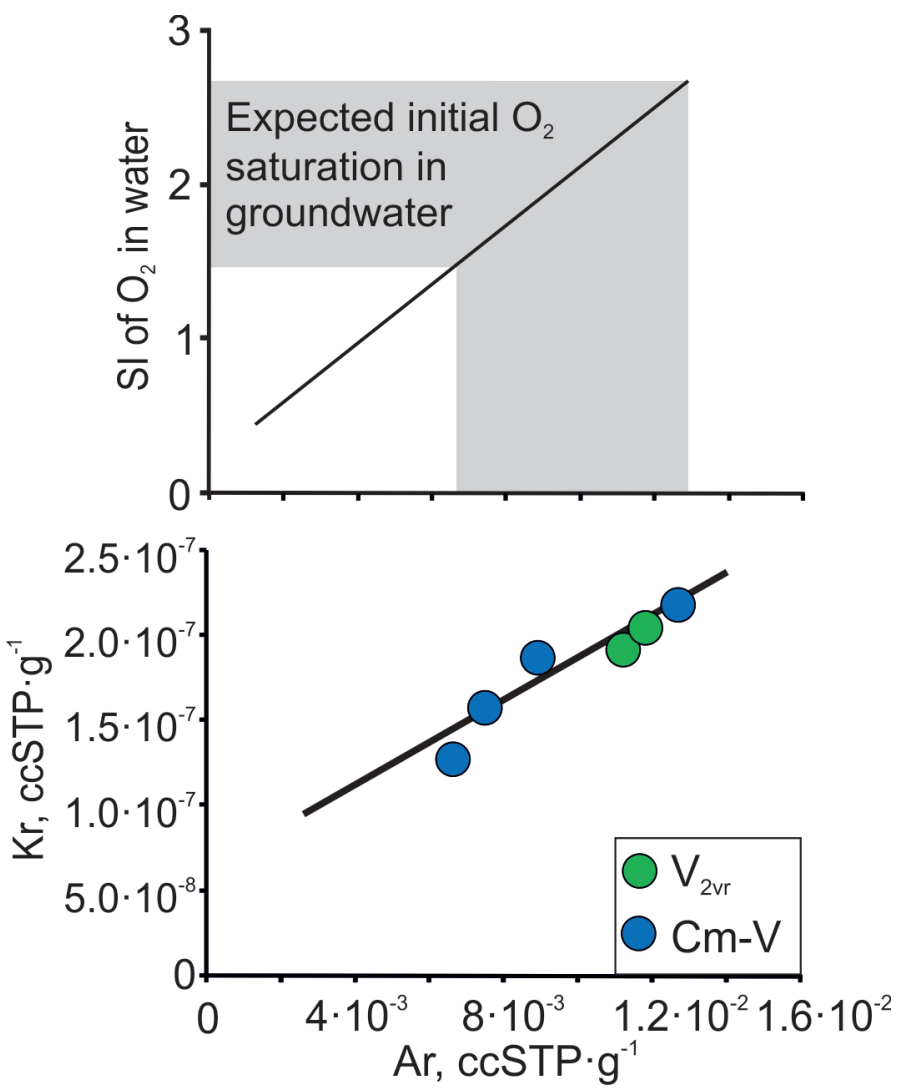

Figure 10. Ar and $\mathrm{Kr}$ contents in $\mathrm{Cm}-\mathrm{V}$ groundwater of the Viimsi peninsula and the expected initial $\mathrm{O}_{2}$ oversaturation in $\mathrm{Cm}-\mathrm{V}$ groundwater at sea level and at $0{ }^{\circ} \mathrm{C}$. The lines follow the trend expected for the addition of air-to-air-equilibrated water.

High dissolved $\mathrm{O}_{2}$ content would have supported $\mathrm{U}$ transport into the aquifer system from the northern direction, where subglacial meltwater would have been in contact with the outcropping crystalline basement rich in U. In aerobic conditions, the U would have become mobile and able to be transported into subsurface by infiltrating glacial meltwater. During the subsequent geochemical evolution of groundwater and the consumption of $\mathrm{O}_{2}$ during the oxidation of pyrite and organic matter [48], the infiltrated glacial meltwater would have evolved towards more reducing conditions, which would have lowered the U solubility. Furthermore, it has been shown that the precipitation of $U$ as uranium carbonate is common near organic-rich clays $[57,59,69,70]$. This would mean that the Cambrian and Ediacaran clays overlying and occurring inside the $\mathrm{Cm}-\mathrm{V}$ aquifer system [54] had provided a suitable environment for $U$ precipitation. We propose a hypothesis that areas near the buried valley southwest of the Viimsi peninsula, where a large section of Lower Cambrian Lontovo clays is cut through by more recent Quaternary sediments, may have been a very suitable site for the development of paleo-redox fronts and for the precipitation of $U$. Thus, ${ }^{226}$ Ra may partly originate from these so-called "secondary $U$ deposits" in the sedimentary complex. The observed pattern in ${ }^{226}$ Ra activity (Figure 9) together with the proposed explanation on its origin would further indicate that the changes in the chemical and isotopic composition of groundwater observed in the Viimsi peninsula are mainly caused by induced recharge of the aquifer system from the buried valley in the south-western part of the peninsula. 
Table 3. Noble gases composition of the $\mathrm{Cm}-\mathrm{V}$ groundwaters in the Viimsi peninsula.

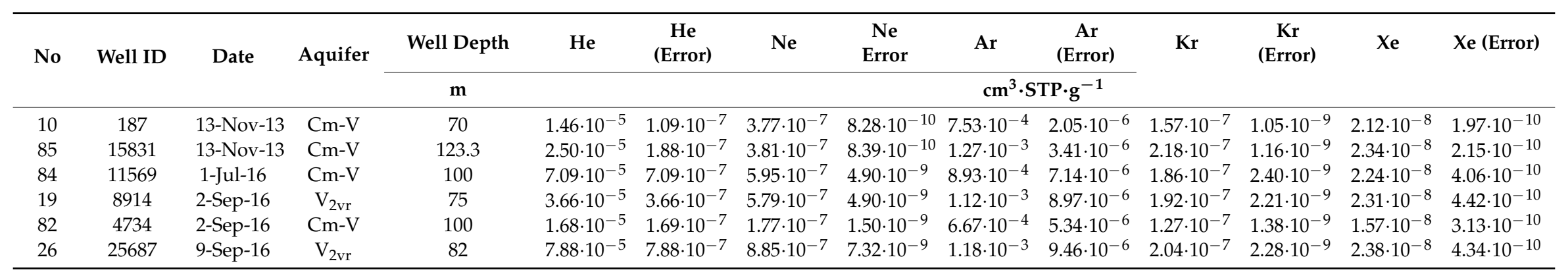




\subsection{Future Prospects}

Salinisation of groundwater resources is a globally significant phenomenon, which results from a high groundwater demand in coastal areas. To avoid saltwater intrusion, sustainable groundwater management strategies are required. However, in the case of the Viimsi peninsula, the salinisation had already taken place in the main groundwater intake before any measures were applied. In order to resolve this situation, a double pumping solution was deployed. This double pumping solution meant that additional groundwater abstraction wells were put into use that were located to the south from the main groundwater intake near the buried valley in the south-western part of the peninsula. This, however, probably facilitated the movement of fresh groundwater from recharge area (buried valley) to the main groundwater intake where intrusion of saline groundwater from the underlying crystalline basement had already occurred. To provide a more precise description of the current situation, a mathematical model of local groundwater dynamics should be developed based on the presented conceptual model. The modelling results would help to optimise groundwater abstraction from different production wells in order to minimise intrusion of brackish water from the underlying crystalline basement. The future management plans for $\mathrm{Cm}-\mathrm{V}$ aquifer system on the Viimsi peninsula would also need to take into account the possibility of surface pollution transported by modern meteoric water from the buried valley reaching the aquifer system. The evolved dual pumping solution and an optimisation model may also be applicable in other areas facing similar problems.

Although we detected no intrusion of seawater in the Viimsi peninsula, its risk in the future cannot be ignored. As the population on the peninsula is increasing, a further rise in water consumption is inevitable and, in the end, the hydraulic head compensation through induced inflow from the crystalline basement and meteoric water infiltration through the buried valley will not be sufficient to avoid seawater intrusion. Most wells in the Viimsi peninsula are 1 to $5 \mathrm{~km}$ away from the outcrop area of the aquifer, and seawater intrusion to the water intake takes time. At the same time, wells in and round the buried valley are practically at the outcrop area (Figure 1), where the aquifer system is most exposed to the intrusion of water from exterior sources.

Predicting the dynamics of future seawater intrusion would require multicomponent strategies that consider intrusion in a broader context on a basin-wide scale and include integrated groundwater and surface water management. The presented conceptual model about chemical changes in the Viimsi peninsula needs a more detailed future analysis, which would include the modelling of transient groundwater dynamics and mass-transport. In addition, more studies on the changes of potentiometric levels and hydrostratigraphy in the Viimsi peninsula are necessary to build reliable solute-transport models based on conservative tracers, such as $\mathrm{Cl}^{-}$and $\delta^{18} \mathrm{O}$.

Accurate three-dimensional characterisation of possible seawater intrusion requires an adequate monitoring network. For the moment, the monitoring wells are in northern and central parts of the peninsula and no wells belonging to the national groundwater-monitoring network are either on or near the buried valley. The available hydrogeological and hydrochemical information from this area can only be attained from private water supplies, which use groundwater from the $\mathrm{Cm}-\mathrm{V}$ aquifer system. This setup is not well suited for studying changes in groundwater quality, as the production wells are located about 3 to $5 \mathrm{~km}$ away from the buried valley. Thus, any changes in groundwater quality imposed by the induced recharge from the buried valley would only be detectable in the production wells long after they have started to operate. Furthermore, the construction of some production wells currently monitored is not ideal for following changes in groundwater quality and quantity, as they open both the deeper Gdov aquifer and the shallower Voronka aquifer.

Additionally, the area is located on the trajectory of the planned tunnel between Tallinn and Helsinki [71]. The future construction of that tunnel would also affect the $\mathrm{Cm}-\mathrm{V}$ aquifer system on the Viimsi peninsula. It is possible that the outcrop area of the $\mathrm{Cm}-\mathrm{V}$ aquifer system that surrounds the Viimsi peninsula is covered by low-porosity sediments like clay or mud, which would prevent seawater intrusion. Thus, it is essential to plan the construction of the tunnel in such a way that the integrity of these aquitards would not be disturbed. 
The groundwater of glacial origin is uncommon in Europe and widespread only in Estonia [72], but such a wide distribution of groundwater with a similar origin as in the $\mathrm{Cm}-\mathrm{V}$ aquifer system has been observed in USA and Canada (Michigan, Alberta and Illinois basins) where they are an important source of drinking water [73-77]. Interpretation of the noble gas results indicates that the groundwater of glacial origin may have been heavily supersaturated with respect to oxygen. In appropriate geological conditions, the accumulation of redox-sensitive elements in the recharge area (e.g., Mn, U) may occur during infiltration of such waters. To know of the presence of such accumulation aids is important for the construction of new water intakes in order to avoid potential natural contamination of water with toxic microcomponents.

\section{Conclusions}

Trends in ${ }^{226 / 228} \mathrm{Ra}, \delta^{18} \mathrm{O}$ and major ions concentrations, in combination with additional hydrochemical information on regional hydrogeology of the $\mathrm{Cm}-\mathrm{V}$ aquifer system from 1987 to 2018, were used to study the causes of the observed water quality changes induced by groundwater abstraction on the Viimsi peninsula near Tallinn, Estonia. Increased groundwater abstraction in the Viimsi area together with the current setup of production wells, distributed in very close proximity from each other in the central part of the peninsula, has led to increased concentrations of $\mathrm{Cl}^{-}$ and enrichment of naturally light isotopic composition of glacial palaeogroundwater in the aquifer system. These changes are caused by mixing between glacial palaeogroundwater with water from the underlying crystalline basement and modern groundwater recharging from the buried valley in the south-western part of the peninsula, due to the lowering of potentiometric levels in the $\mathrm{Cm}-\mathrm{V}$ aquifer system. $\mathrm{Cl}^{-}$concentrations increase because of the upwelling of saline water from the underlying crystalline basement. At the same time, the inflow of meteoric water through a buried valley and the scattering of groundwater production wells away from the central groundwater intake in recent years has lowered the $\mathrm{Cl}^{-}$contents and caused the enrichment in $\delta^{18} \mathrm{O}$, due to wider meteoric water inflow through the buried valley and mixing with saline water.

The changes in groundwater quality have also brought about an increase in Ra activity in the study area. We propose two sources for these increased Ra activities in the aquifer system: The crystalline basement and the secondary $U$ deposits in sedimentary sequence originating from palaeo-redox-front in the south-western part of the peninsula developed in the Pleistocene. The three-component mixing between palaeogroundwater, saline water and meteoric water is the main process controlling the ${ }^{226} \mathrm{Ra}$ activity in the $\mathrm{Cm}-\mathrm{V}$ groundwater. However, the ${ }^{226} \mathrm{Ra}$ activities have possibly also been affected by cation exchange and dissolution/precipitation of the carbonate minerals.

The $\mathrm{Cm}-\mathrm{V}$ aquifer system in the Viimsi peninsula is very vulnerable to overconsumption of groundwater, because it contains essentially non-renewable palaeogroundwater originating from Pleistocene glaciations. Thus, it is critical to understand the causes of the observed changes in water quality for the sustainable management of groundwater resources in the area. Current results give reason to believe that the present groundwater monitoring strategies may not be sufficient to understand and monitor the changes in the $\mathrm{Cm}-\mathrm{V}$ aquifer system in the peninsula. Accurate three-dimensional characterisation of potential seawater intrusion will require increased use of monitoring wells opened at different depths in the south-western part of the peninsula, supplemented by innovative applications of surface and borehole geophysics. In addition, the observed differences in the distribution of ${ }^{226} \mathrm{Ra}$ and ${ }^{228} \mathrm{Ra}$ in groundwater and their relations with the possible paleo-redox fronts and secondary $U$ deposits, could be a topic for wider future research especially in areas where wide distribution of glacial palaeogroundwater has also been observed (e.g. Michigan, Illinois and Alberta Basins in North America).

Author Contributions: Conceptualization, V.R.; validation, W.A., M.K., and R.M.; formal analysis, J.I., G.C., S.S., T.W. and V.S.; writing-original draft preparation, V.R.; writing-review and editing, J.P. and W.A.; visualization, V.R. and S.T. 
Acknowledgments: This research was funded by the Estonian Research Council projects MOBTP25 (V.R.), PUTJD127 (V.R.) and SA Archimedes Project SLOFY12064T (M.K.). We would also like to thank the reviewers for their helpful comments, which made the manuscript clearer and more precise.

Conflicts of Interest: The authors declare no conflict of interest.

\section{Appendix A}

\begin{tabular}{|c|c|}
\hline 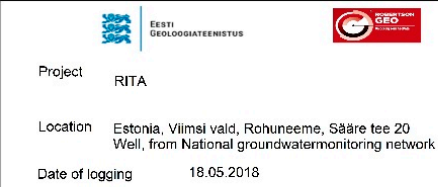 & $\begin{array}{lll}\text { Well number: } & 1151 & \\
\text { Well coordinates: } & \text { Lat } \mathrm{N} & 59,565145 \\
\quad & \text { Long E } & 24,798355 \\
\text { Ground elevation, } \mathrm{m} & 2,8 \\
\text { Depth from ground, } \mathrm{m} & 154,5 \\
\text { Remarks: } & \text { all measurments from ground leve }\end{array}$ \\
\hline LITOLOGY & NATURAL GAMMA \\
\hline Description & API \\
\hline
\end{tabular}

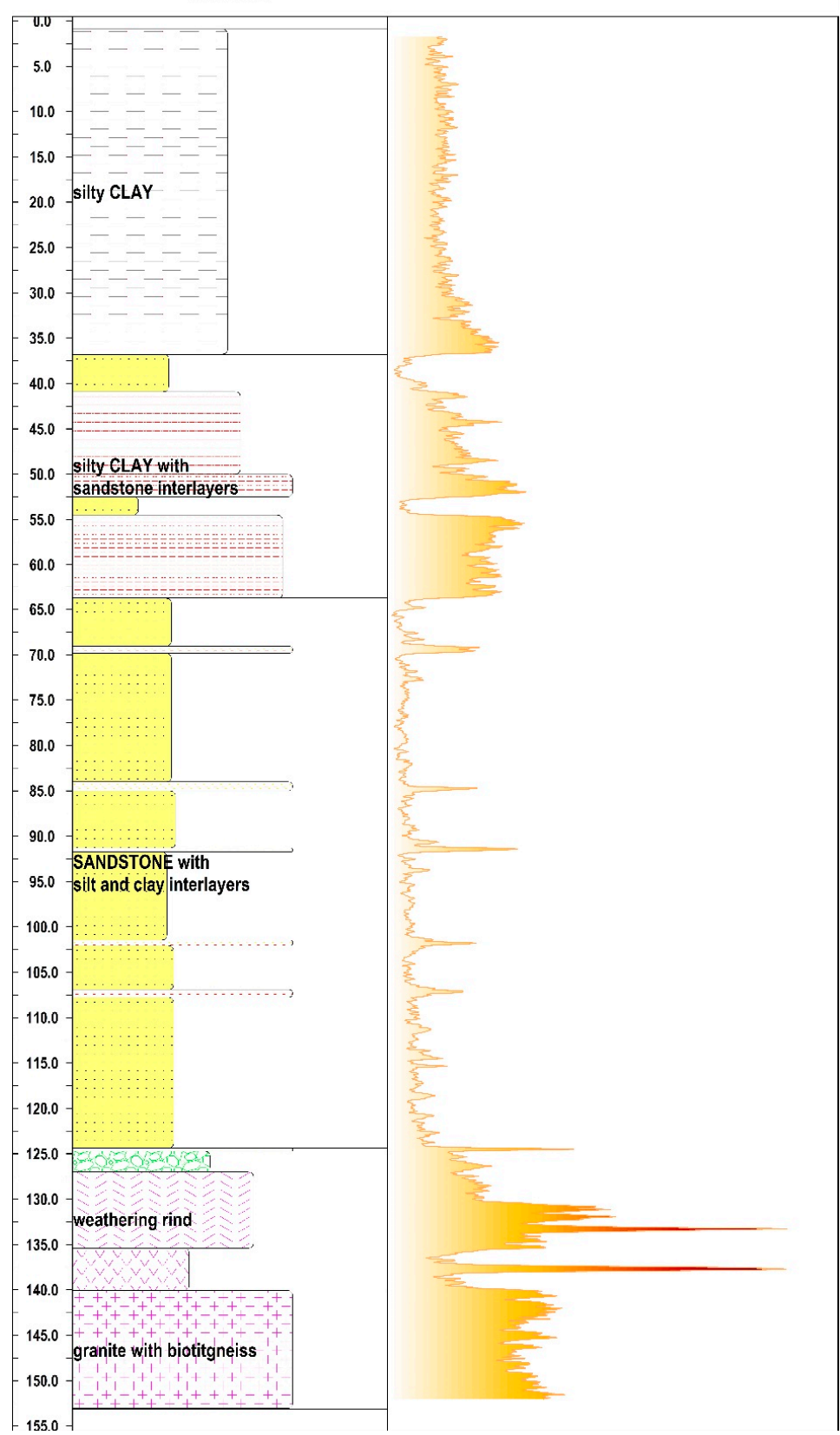

Figure A1. Gamma radiation log from well 1511. 


\section{References}

1. Ferguson, G.; Gleeson, T. Vulnerability of coastal aquifers to groundwater use and climate change. Nat. Clim. Chang. 2012, 2, 342-345. [CrossRef]

2. Werner, A.D.; Bakker, M.; Post, V.E.A.; van den Bohede, A.; Lu, C.; Ataie-Ashtiani, B.; Simmons, C.T.; Barry, D.A. Seawater intrusion processes, investigation and management: Recent advances and future challenges. Adv. Water Resour. 2013, 5, 3-26. [CrossRef]

3. Wetzel, M.; Kühn, M. Salinization of freshwater aquifers due to subsurface fluid injection quantified by species transport simulations. Energy Procedia 2016, 97, 411-418. [CrossRef]

4. Colombani, N.; Osti, A.; Volta, G.; Mastrocicco, M. Impact of climate change on salinization of coastal water resources. Water Resour. Manag. 2016, 30, 2483-2496. [CrossRef]

5. Delfs, J.-O.; Nordbeck, J.; Bauer, S. Upward brine migration resulting from pressure increases in a layered subsurface system. Env. Earth Sci. 2016, 75, 1441. [CrossRef]

6. Vallejos, A.; Sola, F.; Yechieli, Y.; Pulido-Bosch, A. Influence of the paleogeographic evolution on the groundwater salinity in a coastal aquifer. Cabo De Gata Aquiferse Spain J. Hydrol. 2018, 557, 55-66.

7. Edmunds, W.M. Paleowater in European coastal aquifers-The goals and main conclusions of the PALAEAUX project. In Paleowaters in Coastal Europe: Evolution of Groundwater Since the Late Pleistocene; Edmunds, W.M., Milne, C.J., Eds.; Geological Society London Special Publications: London, UK, 2001; pp. 1-16.

8. Cary, L.; Petelet-Giraud, E.; Bertrand, G.; Kloppmann, W.; Aquilina, L.; Martins, V.; Hirata, R.; Montenegro, S.; Pauwels, H.; Chatton, E.; et al. Origins and processes of groundwater salinization in the urban coastal aquifers of Recife (Pernambuco, Brazil): A multi-isotope approach. Sci. Total Environ. 2015, 530-531, 411-429. [CrossRef] [PubMed]

9. Daniele, L.; Vallejos, A.; Corbellá, M.; Molina, L.; Pulido-Bosch, A. Hydrogeochemistry and geochemical simulations to assess water-rock interactions in complex carbonate aquifers: The case of Aguadulce (SE Spain). Appl. Geochem. 2013, 29, 43-54. [CrossRef]

10. Mollema, P.; Antonellini, M.; Dinelli, E.; Gabbianelli, G.; Greggio, N.; Stuyfzand, P. Hydrochemical and physical processes influencing salinization and freshening in mediterranean low-lying coastal environments. Appl. Geochem. 2013, 34, 207-221. [CrossRef]

11. Carreira, P.M.; Marques, J.M.; Nunes, D. Source of groundwater salinity in coastline aquifers based on environmental isotopes (Portugal): Natural vs. human interference-A review and reinterpretation. Appl. Geochem. 2014, 41, 163-175. [CrossRef]

12. Valocchi, A.J.; Street, R.L.; Roberts, P.V. Transport of ion-exchanging solutes in groundwater: Chromatographic theory and field simulation. Water Resour. Res. 1981, 17, 1517-1527. [CrossRef]

13. Appelo, C.A.J. Cation and proton exchange, $\mathrm{pH}$ variations, and carbonate reactions in a freshening aquifer. Water Resour. Res. 1994, 30, 2793-2805. [CrossRef]

14. Appelo, C.A.J.; Willemsen, A. Geochemical calculations and observations on salt water intrusions. I: A combined geochemical/mixing cell model. J. Hydrol. 1987, 94, 313-330. [CrossRef]

15. Van der Kemp, W.; Appelo, C.A.J.; Walraevens, K. Inverse chemical modeling and radiocarbon dating of palaeogroundwaters: The Tertiary Ledo-Paniselian aquifer in Flanders, Belgium. Water Resour. Res. 2000, 36, 1277-1287. [CrossRef]

16. Martinez, D.E.; Bocanegra, E.M. Hydrochemistry and cation-exchange processes in the coastal aquifer of Mar Del Plata, Argentina. Hydrogeol. J. 2002, 10, 393-408. [CrossRef]

17. Giménez, F.E. Dynamic of sea water interface using hydrochemical facies evolution diagram. Ground Water 2010, 48, 212-216. [CrossRef]

18. Raidla, V.; Kirsimäe, K.; Vaikmäe, R.; Jõeleht, A.; Karro, E.; Marandi, A.; Savitskaja, L. Geochemical evolution of groundwater in the Cambrian-Vendian aquifer system of the Baltic Basin. Chem. Geol. 2009, 258, $219-231$. [CrossRef]

19. Raidla, V.; Kern, Z.; Pärn, J.; Babre, A.; Erg, K.; Ivask, J.; Kalvāns, A.; Kohán, B.; Lelgus, M.; Martma, T.; et al. A $\delta^{18} \mathrm{O}$ isoscape for the shallow groundwater in the Baltic Artesian Basin. J. Hydrol. 2016, 542, $254-267$. [CrossRef] 
20. Vaikmäe, R.; Vallner, L.; Loosli, H.H.; Blaser, P.C.; Juillard-Tardent, M. Palaeogroundwater of glacial origin in the Cambrian-Vendian aquifer of northern Estonia. In Palaeowaters of Coastal Europe: Evolution of Groundwater since the late Pleistocene; Edmunds, W.M., Milne, C.J., Eds.; Geological Society: London, UK, 2001; Volume 189, pp. 17-27.

21. Raidla, V.; Kirsimäe, K.; Vaikmäe, R.; Kaup, E.; Martma, T. Carbon isotope systematics of the Cambrian-Vendian aquifer system in the northern Baltic Basin: Implications to the age and evolution of groundwater. Appl. Geochem. 2012, 27, 2042-2052. [CrossRef]

22. Perens, R.; Savitski, L.; Savva, V.; Jaštšuk, S.; Häelm, M. Delineation of Groundwater Bodies and Description of Their Boundaries and Hydrogeological Conceptual Models; Geological Survey of Estonia: Tallinn, Estonia, 2012. (In Estonian)

23. Erg, K.; Truu, M.; Kebbinau, K.; Lelgus, M.; Tarros, S. Report of Estonian Environmental Monitoring. Monitoring of Groundwater Bodies in 2016 of State Environmental Programme; Geological Survey of Estonia: Tallinn, Estonia, 2017. (In Estonia)

24. Suursoo, S.; Hill, L.; Raidla, V.; Kiisk, M.; Jantsikene, A.; Nilb, N.; Czuppon, G.; Putk, K.; Munter, R.; Koch, R.; et al. Temporal changes in radiological and chemical composition of Cambrian-Vendian groundwater in conditions of intensive water consumption. Sci. Total Environ. 2017, 601-602, 679-690. [CrossRef]

25. Savitskaja, L.; Viigand, A. Report of Microcomponent and Isotope Composition Research in Cm-V Aquifer Groundwater for Estimating Drinking Water Quality in North Estonia; Geological Survey of Estonia: Tallinn, Estonia, 1994. (In Estonian)

26. Mokrik, R.; Karro, E.; Savitskaja, L.; Drevaliene, G. The origin of barium in the Cambrian-Vendian aquifer system, North Estonia. Est. J. Earth Sci. 2009, 58, 193-208. [CrossRef]

27. Forte, M.; Bagnato, L.; Caldognetto, E.; Risica, S.; Trotti, F.; Rusconi, R. Radium isotopes in Estonian groundwater: Measurements, analytical correlations, population dose and a proposal for a monitoring stradegy. J. Radiat. Prot. 2010, 30, 761-780. [CrossRef] [PubMed]

28. Vinson, D.S.; Vengosh, A.; Hirschfeld, D.; Dwyer, G.S. Relationships between radium and radon occurrence and hydrochemistry in fresh groundwater from fractured crystalline rocks, North Carolina (USA). Chem. Geol. 2009, 260, 159-171. [CrossRef]

29. Vengosh, A.; Hirschfeld, D.; Vinson, D.; Dwyer, G.; Raanan, H.; Rimawi, O.; Al-Zoubi, A.; Akkawi, E.; Marie, A.; Haquin, G.; et al. High naturally occurring radioactivity in fossil groundwater from the Middle East. Environ. Sci. Technol. 2009, 43, 1769-1775. [CrossRef] [PubMed]

30. Szabo, Z.; DePaul, V.T.; Fischer, J.M.; Kraemer, T.F.; Jacobsen, E. Occurrence and geochemistry of radium in water from principal drinking-water aquifer systems of the United States. Appl. Geochem. 2012, 27, 729-752. [CrossRef]

31. Langmuir, D.; Riese, A.C. The thermodynamic properties of radium. Geochim. Cosmochim. Acta 1985, 49, 1593-1601. [CrossRef]

32. Kraemer, T.F.; Reid, D.F. The occurrence and behavior of radium in saline formation water of the U.S. Gulf Coast region. Chem. Geol. 1984, 2, 153-174. [CrossRef]

33. Sturchio, N.C.; Banner, J.L.; Binz, C.M.; Heraty, L.B.; Musgrove, M. Radium geochemistry of ground waters in Paleozoic carbonate aquifers, midcontinent, USA. Appl. Geochem. 2001, 16, 109-122. [CrossRef]

34. Swarzenski, P.W.; Baskaran, M.; Rosenbauer, R.J.; Edwards, B.D.; Land, M. A combined radio- and stable-isotopic study of a California coastal aquifer system. Water 2013, 5, 480-504. [CrossRef]

35. Sherif, M.I.; Linb, J.; Poghosyan, A.; Abouelmagd, A.; Sultan, M.I.; Sturchio, N.C. Geological and hydrogeochemical controls on radium isotopes in groundwater of the Sinai Peninsula, Egypt. Sci. Total Environ. 2018, 613-614, 877-885. [CrossRef]

36. Porcelli, D.; Swarzenski, P.W. The behavior of U- and Th-series nuclides in groundwater. Rev. Mineral. Geochem. 2003, 52, 317-361. [CrossRef]

37. Vaaramaa, K.; Lehto, J.; Ervanne, H. Soluble and Particle-Bound ${ }^{234,238} \mathrm{U},{ }^{226} \mathrm{Ra}$ and ${ }^{210}$ Po in ground waters. Radiochim. Acta 2003, 91, 21-27. [CrossRef]

38. Vinson, D.S.; Tagma, T.; Bouchaou, L.; Dwyer, G.; Warner, N.R. Occurrence and mobilization of radium in fresh to saline coastal groundwater inferred from geochemical and isotopic tracers. Appl. Geochem. 2013, 38, 161-175. [CrossRef]

39. Perens, R.; Vallner, L. Water-bearing formation. In Geology and Mineral Resources of Estonia; Raukas, A., Teedumäe, A., Eds.; Estonian Academy Publishers: Tallinn, Estonia, 1997; pp. 137-145. 
40. Puura, V.; Vaher, R.; Klein, V.; Koppelmaa, H.; Niin, M.; Vanamb, V.; Kirs, J. Zoning and stratification of the rock complexes of the cristaline basement. In The Crystalline Basement of Estonian Territory; Viiding, H., Ed.; Nauka: Moscow, Russia, 1983; pp. 15-45. (In Russian)

41. Kirs, J.; Puura, V.; Soesoo, A.; Klein, V.; Konsa, M.; Koppelmaa, H.; Niin, M.; Urtson, K. The crystalline basement of Estonia: Rock complexes of the Palaeoproterozoic Orosirian and Statherian and Mesoproterozoic Calymmian periods, and regional correlations. Est. J. Earth Sci. 2009, 58, 219-228. [CrossRef]

42. Karro, E.; Marandi, A.; Vaikmäe, R. The origin of increased salinity in the Cambrian-Vendian aquifer system on the Kopli Peninsula, northern Estonia. Hydrogeol. J. 2004, 12, 424-435. [CrossRef]

43. Savitskaja, L.; Jaštšuk, S. Determination of Radionuclides Concentrations in Groundwater from Cambrian-Vendian Aquifer System and Assessment of Its Conformity to the Norms set by EU Drinking Water Directive 98/83/EC; Geological Survey of Estonia: Tallinn, Estonia, 2001. (In Estonian)

44. Suursoo, S.; Kiisk, M.; Al-Malahmeh, A.; Jantsikene, A.; Lumiste, L. ${ }^{226}$ Ra measurement by lsc as a tool to assess the efficiency of a water treatment technology for removing radionuclides from groundwater. Appl. Radiat. Isot. 2014, 93, 57-63. [CrossRef] [PubMed]

45. Aeschbach-Hertig, W.; Solomon, D.K. Noble gas thermometry in groundwater hydrology. In The Noble Gases as Geochemical Tracers; Burnard, P., Ed.; Springer: Berlin, Germany, 2013; pp. 81-122.

46. Yezhova, M.; Polyakov, V.; Tkachenko, A.; Savitski, L.; Belkina, V. Palaeowaters of North Estonia and their influence on changes of resources and the quality of fresh groundwaters of large coastal water supplies. Geologija 1996, 19, 37-40.

47. Savitski, L.; Viigand, A.; Belkina, V.; Jashtshuk, S. The Estimation of Groundwater Resources in Tallinn. Seeking Groundwater Supplies for Tallinn and Its Vicinity; Geological Survey of Estonia: Tallinn, Estonia, 1993. (In Estonian)

48. Raidla, V.; Kirsimäe, K.; Ivask, J.; Kaup, E.; Knöller, K.; Marandi, A.; Martma, T.; Vaikmäe, R. Sulphur isotope composition of dissolved sulphate in the Cambrian-Vendian aquifer system in the northern part of the Baltic Artesian Basin. Chem. Geol. 2014, 383, 147-154. [CrossRef]

49. Appelo, C.A.J.; Postma, D. Geochemistry, Groundwater and Pollution, 2nd ed.; CRC Press: Boca Raton, FL, USA, 2005.

50. Feistel, R.; Weinreben, S.; Wolf, H.; Seitz, S.; Spitzer, P.; Adel, B.; Nausch, G.; Schneider, B.; Wright, D.G. Density and Absolute Salinity of the Baltic Sea 2006-2009. Ocean Sci. 2010, 6, 3-24. [CrossRef]

51. Arhangelski, B.N.; Fedorova, A.M. Cambrian-Vendian aquifer system. In Hydrogeology in U.S.S.R. XXX; Arhangelski, B.N., Ed.; Nedra: Moscow, Russia, 1966; pp. 170-192. (In Russian)

52. Petersell, V.; Kivisilla, J.; Pukkonen, E.; Põldvere, A.; Täht, K. Evaluation of Ore Events and Mineralization Points in Estonian Bedrock and Crystalline Basement; Geological Survey of Estonia: Tallinn, Estonia, 1991. (In Russian)

53. Raudsep, R. Mineral resources, mineral occurrences. In Geology and Mineral Resources of Estonia; Raukas, A., Teedumäe, A., Eds.; Estonian Academy Publishers: Tallinn, Estonia, 1997; pp. 369-372.

54. Raidla, V.; Kirsimäe, K.; Bityukova, L.; Jõeleht, A.; Shogenova, A.; Šliaupa, S. Lithology and diagenesis of the poorly consolidated Cambrian siliciclastic sediments in the northern Baltic Sedimentary Basin. Geol. Q. 2006, $50,11-22$.

55. Liivamägi, S.; Somelar, P.; Mahaney, W.C.; Kirs, J.; Vircava, I.; Kirsimäe, K. Late Neoproterozoic Baltic paleosol: Intense weathering at high latitude? Geology 2014, 42, 323-326. [CrossRef]

56. Liivamägi, S.; Somelar, P.; Vircava, I.; Mahaney, W.C.; Kirs, J.; Kirsimäe, K. Petrology, mineralogy and geochemical climofunctions of the Neoproterozoic Baltic paleosol. Precambrian Res. 2015, 256, 170-188. [CrossRef]

57. Stackelberg, P.E.; Szabo, Z.; Jurgens, B.C. Radium mobility and the age of groundwater in public-drinking-water supplies from the Cambrian-Ordovician aquifer system, north-central USA. Appl. Geochem. 2018, 89, 34-48. [CrossRef]

58. Swarzenski, P.W. U/Th series radionuclides as tracers of coastal groundwater. Chem. Rev. 2007, 107, 663-674. [CrossRef] [PubMed]

59. Langmuir, D. Aqueous Environmental Geochemistry, 1st ed.; Prentice Hall: New Jersey, NJ, USA, 1997.

60. King, P.T.; Michel, J.; Moore, W.S. Ground water geochemistry of ${ }^{228} \mathrm{Ra},{ }^{226} \mathrm{Ra}$ and ${ }^{222} \mathrm{Rn}$. Geochim. Cosmochim. Acta 1982, 46, 1173-1182. [CrossRef]

61. Chau, N.D.; Lucyna, R.; Jakub, N.; Paweł, J. Radium isotopes in the Polish Outer Carpathian mineral waters of various chemical composition. J. Environ. Radioact. 2012, 112, 38-44. [CrossRef] [PubMed] 
62. Rosholt, J.N. Isotopic composition of uranium and thorium in crystalline rocks. J. Geophys. Res. Solid Earth 1983, 88, 7315-7330. [CrossRef]

63. Langmuir, D. Uranium solution-mineral equilibria at low temperatures with applications to sedimentary ore deposits. Geochim. Cosmochim. Acta 1978, 42, 547-569. [CrossRef]

64. Herczeg, A.L.; Simpson, H.J.; Anderson, R.F.; Tfjer, R.M.; Mathieu, G.G.; Deck, B.L. Uranium and radium mobility in groundwaters and brines within the Delaware basin, southeastern New Mexico, U.S.A. Chem. Geol. 1988, 72, 181-196.

65. Guerrero, J.L.; Vallejos, A.; Ceron, J.C.; Sanchez-Martos, F.; Pulido-Bosch, A.; Bolívar, J.P. U-isotopes and 226Ra as tracers of hydrogeochemical processes in carbonated karst aquifers from arid areas. J. Environ. Radioact. 2016, 158-159, 9-20. [CrossRef]

66. Kipfer, R.; Aeschbach-Hertig, W.; Peters, F.; Stute, M. Noble Gases in Lakes and Groundwaters; Porcelli, D., Ballentine, C.J., Wieler, R., Eds.; Mineralogical Society of America: Washington, DC, USA, 2002; pp. 615-690.

67. Grundl, T.; Magnusson, N.; Brennwald, M.S.; Kipfer, R. Mechanisms of subglacial groundwater recharge as derived from noble gas, ${ }^{14} \mathrm{C}$, and stable isotopic data. Earth Planet. Sci. Lett. 2013, 369-370, 78-85. [CrossRef]

68. Severinghaus, J.P.; Sowers, T.; Brook, E.J.; Alley, R.B.; Michael, L.; Bender, M.L. Timing of abrupt climate change at the end of the Younger Dryas interval from thermally fractionated gases in polar ice. Nature 1998, 391, 141-146. [CrossRef]

69. Gilkeson, R.H.; Cartwright, K.; Cowart, J.B.; Holtzman, R.B. Hydrogeologic and Geochemical Studies of Selected Natural Radioisotopes and Barium in Groundwater in Illinois; Final Technical Completion Report to U.S. Bureau of Reclamation, Project B-108-ILL; University of Illinois at Urbana-Champaign, Water Resources Center: St Paul, MN, USA, 1983.

70. Drake, H.; Suksi, J.; Tullborg, E.-L.; Lahaye, Y. Quaternary redox transitions in deep crystalline rock fractures at the western margin of the Greenland ice sheet. Appl. Geochem. 2017, 76, 196-209. [CrossRef]

71. FinEst Link. 2018. Available online: http://www.finestlink.fi/en/ (accessed on 14 January 2018).

72. Pärn, J. Origin and Geochemical Evolution of Palaeogroundwater in the Northern Part of the Baltic Artesian Basin. Doctoral Thesis, Tallinn University of Technology, Tallinn, Estonia, 2018; p. 226.

73. Clayton, R.N.; Friedman, I.; Graf, D.L.; Mayeda, T.K.; Meents, W.F.; Shimp, N.F. The origin of saline formation waters. Isotopic composition. J. Geophys. Res. 1966, 71, 3869-3882. [CrossRef]

74. Grasby, S.E.; Osadetz, K.; Betcher, R.; Render, F. Reversal of the regional-scale flowsystem of the Williston Basin in response to Pleistocene glaciation. Geology 2000, 29, 635-638. [CrossRef]

75. McIntosh, J.C.; Walter, L.M. Paleowaters in Silurian-Devonian carbonate aquifers: Geochemical evolution of groundwater in the Great Lakes region since the late Pleistocene. Geochim. Cosmochim. Acta 2006, 70, 2454-2479. [CrossRef]

76. Person, M.; McIntosh, J.C.; Remenda, V.; Bense, V. Pleistocene hydrology of North America: The role of ice sheets in reorganizing groundwater systems. Rev. Geophys. 2007, 45, RG3007. [CrossRef]

77. Siegel, D.I.; Mandle, R.J. Isotopic evidence for glacial meltwater recharge to the Cambrian-Ordovician aquifer, north-central United States. Quat. Res. 1984, 22, 328-335. [CrossRef]

(C) 2019 by the authors. Licensee MDPI, Basel, Switzerland. This article is an open access article distributed under the terms and conditions of the Creative Commons Attribution (CC BY) license (http:/ / creativecommons.org/licenses/by/4.0/). 